\title{
ESTIMATING CORRELATED JUMPS AND STOCHASTIC VOLATILITIES
}

\author{
Jiři Witzany*
}

\begin{abstract}
:
We formulate a bivariate stochastic volatility jump-diffusion model with correlated jumps and volatilities. An MCMC Metropolis-Hastings sampling algorithm is proposed to estimate the model's parameters and latent state variables (jumps and stochastic volatilities) given observed returns. The methodology is successfully tested on several artificially generated bivariate time series and then on the two most important Czech domestic financial market time series of the FX (CZK/EUR) and stock (PX index) returns. Four bivariate models with and without jumps and/or stochastic volatility are compared using the deviance information criterion (DIC) confirming importance of incorporation of jumps and stochastic volatility into the model.
\end{abstract}

Keywords: jump-diffusion, stochastic volatility, MCMC, Value at Risk, Monte Carlo

JEL Classification: C11, C15, G1

\section{Introduction}

A number of empirical studies confirmed that financial asset returns are not normal and exhibit fat tails (leptokurtic distribution). Many models going beyond the standard geometrical Brownian diffusion model have been proposed in order to accommodate the empirical facts. The most prominent are jump-diffusion models (see e.g. Cont, Tankov, 2004 for a review), models with stochastic volatility (see Shephard, 2004 for selected papers), or models combining both features, i.e. jump-diffusion models with stochastic volatility, or even models with jumps in volatility. Modelling of portfolio returns or valuation of various multi-asset derivatives requires generalizing of the models into multivariate setting.

We are going to consider a bivariate jump-diffusion model with stochastic volatilities incorporating possible correlation of jump occurrence, jump size, and of stochastic volatilities. The main goal of the paper is to propose an MCMC estimation method that will be tested on artificial and real world data. We have chosen the two most important Czech financial markets time series, namely a series of FX (CZK/EUR) exchange rates and of the stock market (PX index) returns. Joint modelling of the two series might be important for an asset manager exposed to the Czech stock market and the exchange rates.

* University of Economics, Prague, nám. W. Churchilla 4, CZ - 13067 Praha 3 (jiri.witzany@vse.cz). The research has been supported by the Czech Science Foundation Grant No. 402/09/0732 "Market Risk and Financial Derivatives" and by the VŠE Institutional Grant IP 100040. 
The joint return modelling is also important for valuation of quanto-style derivatives. In both cases, the goal is to model the distribution of future returns of a portfolio exposed to both factors.

For example, inspecting the development of the markets in 2004-2011 (Figure 4 - Figure 6) it seems that the returns of the time series exhibit many jumps and periods of low volatility that are followed by periods of high volatility or vice versa. Although the sample correlation of returns turns out to be almost zero, the volatilities appear to move in the same direction (Figure 6). The questions are: How do the two markets jump and how volatile are the stochastic volatilities? Is the non-Gaussian behaviour explained by jumps or rather by stochastic volatility? Are the jumps in the two markets correlated in terms of occurrence and size? And moreover, are the stochastic volatilities correlated?

Our estimation methodology is based on the MCMC (Markov Chain Monte Carlo) approach following Jacquier et al. (2007) and Johannes, Polson (2009). The first breakthrough application of the Bayesian methods for the analysis of stochastic volatility models has been made in Jacquier et al. (1994). The authors applied an MCMC algorithm to estimate parameters as well the latent states of the stochastic volatility model on the US stock return data. The estimation method is shown to outperform other well-known estimation approaches, such as the Method of Moments or the Quasi-Maximum Likelihood Estimator. Since then extensive research on applications of Bayesian methods to stochastic volatility models have appeared (see e.g. Shephard, 2004). Johannes, Kumar (1999) estimate state dependent jump models (on US stock data) in which arrival intensity and jump sizes depend on a given state variable including lagged jumps. Eraker et al. (2003) examine stochastic volatility models incorporating jumps in returns and volatility using US stock indices returns. Eraker (2004) utilizes in addition stock index option date and allows the diffusion and volatility processes being correlated. A related Bayesian Kalman filter approach has been also applied in Šopov, Seidler (2010) in order to study common factors of CZK, HUF, PLN, and SKK yield curve dynamics.

The contribution of this paper is in specification and estimation of a bivariate model with correlated stochastic volatilities and jumps. Johannes, Polson (2009) consider a multivariate version of Merton's jump-diffusion model where jumps occur at the same times for all processes and the jump sizes have a multivariate distribution. Inspecting the times of probable jumps from the MCMC estimation of two univariate jump-diffusion models applied to the two considered return series, we have note the jump times overlap only partially. So, in our specification we have two correlated Poisson processes and correlated jump sizes (if the jumps occur at the same time). Regarding the correlation of stochastic volatilities, we may again firstly inspect the mean stochastic volatilities given by the MCMC algorithm applied to the two processes separately. Since the stochastic volatility residuals do not indicate any significant correlation but show a strong correlation in levels of the stochastic volatilities we propose a bivariate stochastic volatility jump-diffusion model with possible Granger causality between the stochastic 
volatilities as our full model specification (see Asai et al., 2006 or Yu, Meyer, 2006 for an overview of multivariate stochastic volatility models). The computationally difficult estimation of the model is based on our generalization of the method proposed firstly in Jacquier et al. (1994).

The proposed bivariate jump-diffusion with stochastic volatilities will be firstly tested on artificially generated data. The goal of the test is to demonstrate that the estimation procedure yields acceptable results with respect to the generating parameters, in particular that it is able to identify existence of jumps, stochastic volatilities, and their correlations.

The methodology will be finally applied to estimate and compare four bivariate models on the FX and stock returns series, specifically: the ordinary diffusion model, the jump-diffusion model, the diffusion model with stochastic volatility, and the jump-diffusion model with stochastic volatility. Performance of the models will be compared using the deviance information criterion (DIC) generalizing, according to Spiegelhalter et al. (2002), the Akaike information criterion (AIC), and applicable to models with a large number of latent state variables. Importance of the choice of an appropriate model will be illustrated calculating VaR for various time horizons and confidence levels.

\section{Methodology}

In this section we are going to give a brief overview of the relevant stochastic models. We also outline the key elements of the MCMC methods and their implementation in case of the stochastic models under consideration. Finally we shortly describe the classical and Bayesian VaR estimation methodology.

\subsection{Stochastic Asset Price Models}

The most traditional (related to the Black-Scholes formula) continuous-time financial model is the geometric Brownian motion described by the stochastic differential equation (SDE)

$$
d S=\mu S d t+\sigma S d z
$$

where $S(t)$ is an asset price, $\mu$ its drift, $\sigma$ its volatility, and $d z$ the Wiener process increment (see e.g. Shreve, 2004). The equation can be simplified applying the Ito's lemma and the $\log$ transformation into a generalized Wiener process equation

$$
d(\log S)=\left(\mu-\sigma^{2} / 2\right) d t+\sigma d z .
$$

The left-hand side of (2) can be interpreted as the log return over a time interval of the length $d t$. In practice, a time (Euler) discretization is used in order estimate the parameters from an observed financial time series, and in order to generate future returns. In case of the equation the Euler discretization takes the simple form 


$$
r_{i}=\tilde{\mu}+\tilde{\sigma} \epsilon_{i}, \epsilon_{i} \sim N(0,1)
$$

where $r_{i}=\log S_{i} / S_{i-1}$ is the $\log$ return over a regular time interval of length $\Delta t$, $\tilde{\mu}=\left(\mu-\sigma^{2} / 2\right) \Delta t$, and $\tilde{\sigma}=\sigma \sqrt{\Delta t}$. According to (3), observed returns should have a normal distribution. However, many studies demonstrate that the returns have, typically, a leptokurtic or skewed distribution, in particular fat tails. Consequently, the geometric Brownian motion is proposed to be generalized in various directions, in particular, allowing for jumps (see Cont, Tankov, 2004) and stochastic volatility (see Shephard, 2004).

The jump-diffusion SDE can be written in the log-return form as

$$
d(\log S)=\left(\mu-\sigma^{2} / 2-\lambda \mu_{J}\right) d t+\sigma d z+d J
$$

where the jump term, $d J=Z d N$, has a normally distributed $N\left(\mu_{J}, \sigma_{J}\right)$ jump-size component and a component given by the Poisson counting process $N$ with intensity $\lambda$. Essentially, this component adds a mass to the tails of the returns distribution. We consider a time discretization where at most one jump can happen over a single time step:

$$
\begin{aligned}
r_{i} & =\tilde{\mu}+\tilde{\sigma} \epsilon_{i}+Z_{i} J_{i} \\
\epsilon_{i} & \sim N(0,1), Z_{i} \sim N\left(\mu_{J}, \sigma_{J}\right), J_{i} \sim \operatorname{Bern}(\lambda) .
\end{aligned}
$$

According to Eraker et al. (2003) this assumption does not introduce any bias in the parameter estimates.

Stochastic volatility models allow the variance $V=\sigma^{2}$ or log-variance to evolve according to an SDE, i.e. the constant volatility $\sigma$ in (2) or (4) is replaced by the stochastic volatility $\sqrt{ } V$. For example the Heston's (1993) model sets

$$
d V=\kappa(\theta-V) d t+\sigma_{V} \sqrt{V} d z_{V}
$$

Following Jacquier et al. (1994) and Johannes, Polson (2009) will we rather consider the log-variance SDE:

$$
d \log V=\kappa(\theta-\log V) d t+\sigma_{V} d z_{V}
$$

In the discrete setting, with $h_{i}=\log V_{i}$, the equation takes the form of an $\operatorname{AR}(1)$ model:

$$
h_{i}-h_{i-1}=\kappa\left(\theta-h_{i-1}\right) \Delta t+\sigma_{V} \sqrt{\Delta t} \epsilon_{i}^{V}, \epsilon_{i}^{V} \sim N(0,1) .
$$

Rearranging the terms the equation can be written as

$$
h_{i}=\alpha+\beta h_{i-1}+\gamma \epsilon_{i}^{V}
$$

where $\alpha=\kappa \theta \Delta t, \beta=1-\kappa \Delta t$, and $\gamma=\sigma_{V} \sqrt{\Delta t}$. 
If two or more financial assets is to be modelled, then possible correlations needs to be considered. For example $S_{1}$ if and $S_{2}$ are two asset prices following (1) with appropriate indexes we have to admit possibility of correlated $d z_{1}$ and $d z_{2}$. If the prices follow the jump-diffusion process (4) then we have to admit a correlation between $d J_{1}$ and $d J_{2}$. Finally, in case of stochastic volatility (6) we might consider a correlation between $d z_{1}^{V}$ and $d z_{2}^{V}$, but also a mutual correlation between $d z_{1}$ and $d z_{1}^{V}$, and the correlation between $d z_{2}$ and $d z_{2}^{V}$. Due to the mean-reverting form of (6) we should also consider a possible correlation between $\log V_{1}$ and $\log V_{2}$ that could be captured introducing $\log V_{2}$ into the $\mathrm{SDE}$ for $\log V_{1}$ and vice versa. A nonzero coefficient is then interpreted as Granger causality form one asset variance to another.

An analyst that needs to model the distribution of future returns of a portfolio, or of a derivative payoff depending on two or even more assets, stands in front of a difficult task: to choose an optimal model and at the same time to estimate in a feasible way its parameters using historical or currently observable data. Due to increasing complexity of the models we will focus on the MCMC Bayesian estimation and a Bayesian model comparison approach.

\subsection{Markov Chain Monte Carlo (MCMC)}

The Bayesian MCMC sampling algorithm has become a strong and frequently used tool to estimate complex models with multidimensional parameter vectors, including latent state variables. Examples are financial stochastic models with jumps, stochastic volatility processes, models with complex correlation structure, or switching-regime processes. For a more complete treatment of MCMC methods and applications we refer reader for example to Johannes, Polson (2009), Rachev et al. (2008), or Lynch (2007).

MCMC provides a method of sampling from multivariate densities that are not easy to sample from directly, by breaking these densities down into more manageable univariate or lower dimensional multivariate densities. To estimate a vector of unknown parameters $\Theta=\left(\theta_{1}, \ldots, \theta_{k}\right)$ from a given dataset, where we are able to write down the Bayesian marginal densities $p\left(\theta_{j} \mid \theta_{i}, i \neq j\right.$, data $)$ but not the multivariate density $p(\Theta \mid$ data $)$, the MCMC Gibbs sampler works according to the following generic procedure:

0 . Assign a vector of initial values to $\Theta^{0}=\left(\theta_{1}^{0}, \ldots, \theta_{k}^{0}\right)$ and $\operatorname{set} j=0$.

1. Set $j=j+1$.

2. Sample $\theta_{1}^{j} \sim p\left(\theta_{1} \mid \theta_{2}^{j-1}, \ldots, \theta_{k}^{j-1}\right.$, data $)$.

3. Sample $\theta_{2}^{j} \sim p\left(\theta_{2} \mid \theta_{1}^{j}, \theta_{3}^{j-1}, \ldots, \theta_{k}^{j-1}\right.$, data $)$.

$\mathrm{k}+1$. Sample $\theta_{k}^{j} \sim p\left(\theta_{k} \mid \theta_{1}^{j}, \theta_{2}^{j}, \ldots, \theta_{k-1}^{j}\right.$, data $)$ and return to step 1 . 
According to the Clifford-Hammersley theorem the conditional distributions $p\left(\theta_{j} \mid \theta_{i}, i \neq j\right.$, data $)$ fully characterize the joint distribution and moreover under mild conditions the Gibbs sampler distribution converges to the target joint distribution (Johannes, Polson, 2009).

The conditional probabilities are typically obtained applying the Bayes theorem to the likelihood function and a prior density, e.g.

$$
p\left(\theta_{1} \mid \theta_{2}^{j-1}, \ldots, \theta_{k}^{j-1}, \text { data }\right) \propto L\left(\text { data } \mid \theta_{1}, \theta_{2}^{j-1}, \ldots, \theta_{k}^{j-1}\right) \cdot \operatorname{prior}\left(\theta_{1} \mid \theta_{2}^{j-1}, \ldots, \theta_{k}^{j-1}\right) .
$$

We will often use uninformative priors, i.e. $\operatorname{prior}\left(\theta_{i}\right) \propto 1$ and assume that the parameters are independent. In order to apply the Gibbs sampler the right hand side of the proportional relationship needs to be normalized, i.e. we need to be able to integrate the right hand side with respect to $\theta_{1}$ conditional on $\theta_{2}^{j-1}, \ldots, \theta_{k}^{j-1}$.

Useful Gibbs sampling distributions are univariate or multivariate normal, Inverse Gamma $^{1}$ or Wishart, and the Beta distribution. If $\mathbf{y}=\left\langle y_{1}, \ldots, y_{T}\right\rangle$ is an observed series and assuming that iid $y_{i} \sim N\left(\mu, \sigma^{2}\right)$ with unknown parameters $\mu$ and $\sigma$ then

$$
\begin{aligned}
& p(\mu \mid \mathbf{y}, \sigma) \propto L\left(\mathbf{y} \mid \mu, \sigma^{2}\right) p(\mu)=\prod_{i=1}^{T} \varphi\left(y_{i} ; \mu, \sigma\right) \propto\left(\frac{1}{\sqrt{2 \pi} \sigma}\right)^{T} \exp \left(-\frac{\sum\left(y_{i}-\mu\right)^{2}}{2 \sigma^{2}}\right) \\
& \propto \exp \left(-\frac{T \mu^{2}-2 \mu \sum y_{i}}{2 \sigma^{2}}\right) \propto \varphi\left(\mu ; \frac{\sum y_{i}}{T}, \frac{\sigma}{\sqrt{T}}\right)
\end{aligned}
$$

using the uninformative prior $p(\mu) \propto 1$. Moreover

$$
\begin{aligned}
& p\left(\sigma^{2} \mid \mathbf{y}, \mu\right) \propto L\left(\mathbf{y} \mid \mu, \sigma^{2}\right) \cdot p\left(\sigma^{2}\right)=\frac{1}{\sigma^{2}} \prod_{i=1}^{T} \varphi\left(y_{i} ; \mu, \sigma\right) \\
& \propto\left(\sigma^{2}\right)^{-\frac{T}{2}-1} \exp \left(-\frac{\sum\left(y_{i}-\mu\right)^{2}}{2 \sigma^{2}}\right) \propto I G\left(\sigma^{2} ; \frac{T}{2}, \frac{\sum\left(y_{i}-\mu\right)^{2}}{2}\right)
\end{aligned}
$$

using the prior $p\left(\sigma^{2}\right) \propto 1 / \sigma^{2}$ equivalent to the uninformative log-variance prior $p\left(\log \sigma^{2}\right) \propto 1$. Hence the Bayesian distributions for $\mu$ and $\sigma$ can be obtained by the Gibbs sampler iterating (9) and (10). The prior distributions are often specified in order to improve convergence but not to influence (significantly) the final results, typically a wide normal distribution conjugate prior distribution for $\mu$ and a flat inverse gamma distribution for $\sigma^{2}$ are used.

1 Inverse gamma probability distribution density function with the shape parameter $\alpha$ and scale parameter $\beta$ is given by $I G(x ; \alpha, \beta)=\frac{\beta^{\alpha}}{\Gamma(\alpha)} x^{-\alpha-1} \exp (-\beta / x)$ where is the Gamma function. The mean of $x$ is $\mu=\frac{\beta}{\alpha-1}$ for and the variance is $\sigma^{2}=\frac{\beta^{2}}{(\alpha-1)^{2}(\alpha-2)}$ for $\alpha>2$. Alternatively, given $\mu$ and $\sigma^{2}$ we get $\alpha=\frac{\mu^{2}}{\sigma^{2}}+2$ and $\beta=\mu\left(\frac{\mu^{2}}{\sigma^{2}}+1\right)$. 
If the series is multivariate normal then the distributions are generalized to multivariate normal and inverse Wishart (Lynch, 2007). A multivariate discrete-time diffusion process is in fact equivalent to a multivariate normal return series model with iid $\mathbf{r}_{i} \sim N(\boldsymbol{\mu}, \Sigma)$, where $\mathbf{r}_{i}=\left(r_{i, 1}, \ldots, r_{i, m}\right)^{\prime}$ is a vector of returns on $m$ assets observed at time $i, \boldsymbol{\mu}$ is a vector of means, and $\Sigma$ a covariance matrix. The marginal distributions are

$$
p(\boldsymbol{\mu} \mid \mathbf{r}, \Sigma)=\varphi\left(\boldsymbol{\mu} ; \frac{1}{T} \sum_{i=1}^{T} \mathbf{r}_{i}, \frac{1}{T} \Sigma\right)
$$

and

$$
p(\Sigma \mid \mathbf{r}, \boldsymbol{\mu})=I W(\Sigma ; T, S) \propto|\Sigma|^{-(T+m+1) / 2} \exp \left(-\frac{1}{2} \operatorname{tr}\left(\mathrm{S} \Sigma^{-1}\right)\right)
$$

Where $I W(\Sigma ; T, S)$ denotes the inverse Wishart distribution, $S=\sum_{i=1}^{T}\left(\mathbf{r}_{i}-\boldsymbol{\mu}\right)^{\prime}\left(\mathbf{r}_{i}-\boldsymbol{\mu}\right)$ is the scale matrix, and the improper prior $p(\Sigma) \propto|\Sigma|^{-\frac{m+1}{2}}$ analogous to the univariate case has been used.

If $\mathbf{b}=\left\langle b_{1}, \ldots, b_{T}\right\rangle$ is a binary series where $b_{i} \sim \operatorname{Bern}(\lambda)$ iid, then $\lambda$ can be sampled using the beta ${ }^{2}$ distribution:

$$
p(\lambda \mid \mathbf{b}) \propto L(\mathbf{b} \mid \lambda) \cdot p(\lambda) \propto \prod_{i=1}^{T} \lambda^{b_{i}}(1-\lambda)^{1-b_{i}} \propto \lambda^{n}(1-\lambda)^{T-n} \propto \operatorname{Beta}(\lambda ; n+1, T-n+1)
$$

with the uninformative prior $p(\lambda) \propto 1$. Generally, the beta distribution Beta $(x ; \alpha, \beta)$ would be a conjugate prior where $\alpha$ and $\beta$ can be interpreted as prior "successes" and "failures."

If the integration on the right hand side of (8) is not analytically possible (which will be also our case) then the Metropolis-Hastings algorithm can be used. It is based on a rejection sampling algorithm. For example in step 2 the idea is firstly to sample a new proposal value of $\theta_{1}^{j}$ and then accept it or reject it (i.e. reset $\theta_{1}^{j}:=\theta_{1}^{j-1}$ ) with appropriate probability so that, intuitively speaking, we rather move to the parameter estimates with higher corresponding likelihood values.

Specifically, step 1 is replaced with a two step procedure:

1. A. Draw $\theta_{1}^{j}$ from a proposal density $q\left(\theta_{1} \mid \theta_{1}^{j-1}, \theta_{2}^{j-1}, \ldots, \theta_{k}^{j-1}\right.$, data $)$,

B. Accept $\theta_{1}^{j}$ with the probability $\alpha=\min (R, 1)$, where

$$
R=\frac{p\left(\theta_{1}^{j} \mid \theta_{2}^{j-1}, \ldots, \theta_{k}^{j-1}, \text { data }\right) q\left(\theta_{1}^{j-1} \mid \theta_{1}^{j}, \theta_{2}^{j-1}, \ldots, \theta_{k}^{j-1}, \text { data }\right)}{p\left(\theta_{1}^{j-1} \mid \theta_{2}^{j-1}, \ldots, \theta_{k}^{j-1}, \text { data }\right) q\left(\theta_{1}^{j} \mid \theta_{1}^{j-1}, \theta_{2}^{j-1}, \ldots, \theta_{k}^{j-1}, \text { data }\right)} .
$$

In practice the step $1 \mathrm{~B}$ is implemented by sampling a $u \sim U(0,1)$ from the uniform distribution $\theta_{1}^{j}$ and accepting if and only if $u<R$.

2 The pdf of the beta distribution is $\operatorname{Beta}(x ; \alpha, \beta)=\frac{\Gamma(\alpha+\beta)}{\Gamma(\alpha) \Gamma(\beta)} x^{\alpha-1}(1-x)^{\beta-1}$ for $0<\mathrm{x}<1$. The mean can be expressed as $\mu=\frac{\alpha}{\alpha+\beta}$ and the variance $\sigma^{2}=\frac{\alpha \beta}{(\alpha+\beta)^{2}(\alpha+\beta+1)}$. 
It is again shown (see Johannes, Polson, 2009) that under certain mild conditions the limiting distribution is the joint distribution $p(\Theta \mid$ data $)$ of the parameter vector. Note that the limiting distribution does not depend on the proposal density, or on the starting parameter values. The proposal density and the initial estimates only make the algorithm more-or-less numerically efficient.

A popular proposal density is the random walk, i.e. sampling by

$$
\theta_{1}^{j} \sim \theta_{1}^{j-1}+N(0, c) .
$$

The algorithm is then called Random Walk Metropolis-Hastings. The proposal density is in this case symmetric, i.e. the probability of going from $\theta_{1}^{j-1}$ to $\theta_{1}^{j}$ is the same as the probability of going from $\theta_{1}^{j}$ to $\theta_{1}^{j-1}$ (fixing the other parameters), and so the second part of the fraction in the formula (14) for $\alpha$ in step 1B cancels out. Consequently, assuming non-informative prior, the acceptance or rejection is driven just by the likelihood ratio

$$
R=\frac{L\left(\text { data } \mid \theta_{1}^{j}, \theta_{2}^{j-1}, \ldots, \theta_{k}^{j-1}\right)}{L\left(\text { data } \mid \theta_{1}^{j-1}, \theta_{2}^{j-1}, \ldots, \theta_{k}^{j-1}\right)} .
$$

Another popular approach we shall use is the Independence Sampling MetropolisHastings algorithm where the proposal density $\mathrm{q}\left(\theta_{1}^{j}\right)$ does not depend on $\theta_{1}^{j-1}$ (given the other parameters). The acceptance probability ratio (14) is slightly simplified but the proposal densities do not cancel out. In order to achieve efficiency the shape of the proposal density $q$ should be close to the shape of the target density $p$, which is known only up to a normalizing constant.

Typically, estimating complex stochastic models, we need to estimate the parameter vector with a few model parameters $\Theta$, and a vector with a large number of state variables $X$ (proportional to the number of observations). We know that $p(\Theta, X \mid$ data $) \propto p($ data $\mid \Theta, X) \cdot p(X, \Theta)$ and so we may estimate iteratively the parameters and the state variables:

$$
\begin{aligned}
& p(\Theta \mid X, \text { data }) \propto p(\text { data } \mid \Theta, X) \cdot p(X \mid \Theta) \cdot p(\Theta), \\
& p(X \mid \Theta, \text { data }) \propto p(\text { data } \mid \Theta, X) \cdot p(\Theta \mid X) \cdot p(X) .
\end{aligned}
$$

The parameters and state variables are sampled step by step, or in blocks, often combining Gibbs and Metropolis-Hastings sampling.

\subsection{Univariate Jump-diffusion Stochastic Volatility Model}

The main goal of this section is to propose an MCMC estimation algorithm for a bivariate jump diffusion stochastic volatility model. However, it will be useful to outline firstly the univariate jump-diffusion model and the extended model with stochastic volatility. It is then easier to define the sampling steps of the bivariate model estimation algorithm. Moreover, given two financial series, it is useful to estimate firstly the univariate 
models separately on the two series. Since the latent variables, i.e. jumps and stochastic volatilities, are also estimated, the output can be used to make a preliminary analysis of correlations between the jumps and stochastic volatilities.

Let us firstly consider the discrete-time jump diffusion model with constant volatility. Given a sequence of observed returns, the parameters and latent state variables to be estimated are: $\mu, \sigma, \lambda, \mu_{j}, \sigma_{j}, \mathbf{Z}, \mathbf{J}$.

In this case we may use the pure Gibbs MCMC algorithm:

1. Sample reasonable initial values $\mu^{(0)}, \sigma^{(0)}, \lambda^{(0)}, \mu_{J}^{(0)}, \sigma_{J}^{(0)}, \mathbf{Z}^{(0)}, \mathbf{J}^{(0)}$.

2. For $i=1 \ldots, T$ sample $Z_{i}^{(g)} \propto \varphi\left(Z ; \mu_{J}^{(g-1)}, \sigma_{J}^{(g-1)}\right)$ if $J_{i}^{(g-1)}=0$, and

$$
Z_{i}^{(g)} \propto \varphi\left(r_{i} ; \mu^{(g-1)}+Z, \sigma^{(g-1)}\right) \varphi\left(Z ; \mu_{J}^{(g-1)}, \sigma_{J}^{(g-1)}\right) \text { if } J_{i}^{(g-1)}=1 .
$$

3. For $i=1 \ldots, T$ sample $J_{i}^{(g)} \in\{0,1\}, \operatorname{Pr}[J=1]=p_{1} /\left(p_{0}+p_{1}\right)$, where

$$
\begin{aligned}
& p_{0}=\varphi\left(r_{i} ; \mu^{(g-1)}, \sigma^{(g-1)}\right)\left(1-\lambda^{(g-1)}\right), \\
& p_{1}=\varphi\left(r_{i} ; \mu^{(g-1)}+Z, \sigma^{(g-1)}\right) \lambda^{(g-1)}
\end{aligned}
$$

4. Sample $\mu^{(g)}, \sigma^{(g)}$ based on the normally distributed time series $r_{i}-Z_{i}^{(g)} J_{i}^{(g)}$ according to (9) and (10).

5. Sample $\lambda^{(0)}$ based on Bernoulli distributed $J_{i}^{(\mathrm{g})}$ binary time series according to (13).

6. Sample $\mu_{J}^{(g)}, \sigma_{J}^{(g)}$ based on the normally distributed time series $Z_{i}^{(g)}$ according to (9) and (10).

Secondly, let us consider a jump-diffusion model with stochastic volatility following the equation (6). The discrete time specification is:

$$
\begin{aligned}
& r_{i}=\mu+\sqrt{V_{i}} \epsilon_{i}+Z_{i} J_{i} \\
& \log V_{i}=\alpha+\beta \log V_{i-1}+\gamma \epsilon_{i}^{V} \\
& \epsilon_{i}, \epsilon_{i}^{V} \sim N(0,1), Z_{i} \sim N\left(\mu_{J}, \sigma_{J}\right), J_{i} \sim \operatorname{Bern}(\lambda), \text { iid }
\end{aligned}
$$

In this case we need to estimate not only the latent state variables $\mathbf{Z}, \mathbf{J}$ but also the vector of latent stochastic variances $\mathbf{V}$. The MCMC estimation unfortunately requires application of the Metropolis-Hastings since the conditional distribution for the variance $V_{i}$ (conditional on the other variances and parameters) is not a known one. It follows from (16) and the Bayes Theorem that:

$$
p\left(V_{i} \mid \mathbf{V}_{(-i)}, \Theta, \mathbf{r}, \mathbf{Z}, \mathbf{J}\right) \propto p\left(r_{i} \mid V_{i}, \Theta, Z_{i}, J_{i}\right) p\left(V_{i} \mid V_{i-1}, \Theta\right) p\left(V_{i+1} \mid V_{i}, \Theta\right) .
$$

Here the first part of the right hand side of (17) is inverse gamma in $V_{i}$ :

$$
p\left(r_{i} \mid V_{i}, \Theta, Z_{i}, J_{i}\right)=\varphi\left(r_{i} ; \mu+Z_{i} J_{i}, V_{i}\right) \propto V_{i}^{-0.5} \exp \left(-0.5\left(r_{i}-\mu-Z_{i} J_{i}\right)^{2} / V_{i}\right) .
$$


But the remaining two factors are lognormal ${ }^{3}$ in $V_{i}$ :

$$
\begin{gathered}
\log V_{i} \sim \varphi\left(\log V_{i} ; \alpha+\beta \log V_{i-1}, \gamma\right), \text { i.e. } \\
p\left(V_{i} \mid V_{i-1}, \Theta\right) \propto V_{i}^{-1} \exp \left(-\left(\log V_{i}-\alpha-\beta \log V_{i-1}\right)^{2} /\left(2 \gamma^{2}\right)\right),
\end{gathered}
$$

and similarly

$$
\begin{gathered}
\log V_{i+1} \sim \varphi\left(\log V_{i+1} ; \alpha+\beta \log V_{i}, \gamma\right) \text {, i.e. } \\
p\left(V_{i+1} \mid V_{i}, \Theta\right) \propto \exp \left(-\left(\log V_{i+1}-\alpha-\beta \log V_{i}\right)^{2} /\left(2 \gamma^{2}\right)\right) \text { in terms of } V_{i} .
\end{gathered}
$$

It is easy to verify that the product of the two lognormal distributions is proportional to the lognormal distribution with the corresponding normal distribution mean and standard deviation:

$$
\begin{aligned}
& \mu_{i}=\left(\alpha(1-\beta)+\beta\left(\log V_{i+1}+\log V_{i-1}\right)\right) /\left(1+\beta^{2}\right), \\
& \sigma=\gamma / \sqrt{1+\beta^{2}} .
\end{aligned}
$$

In order to obtain a proposal distribution Jacquier et al (1994) suggest replacing the lognormal distribution with an inverse gamma distribution fitting the first two moments. It is confirmed empirically that the choice of a proposal distribution with a shape closely mimicking the original distribution is of key importance, since high dimensionality of the variance state variable vector makes convergence of the MCMC algorithm difficult.

The product of two inverse gamma distribution density functions is an inverse gamma distribution density function, hence combining the inverse gamma distribution (18) and the fitted inverse gamma distribution we finally obtain the proposal density function:

$$
\begin{aligned}
& q\left(V_{i} \mid \mathbf{V}_{(-i)}, \Theta, \mathbf{r}, \mathbf{Z}, \mathbf{J}\right)=I G\left(V_{i} ; \phi+0,5,(\phi-1) \exp \left(\mu_{i}+0.5 \sigma^{2}\right)+0.5\left(r_{i}-\mu-Z_{i} J_{i}\right)^{2}\right), \\
& \text { where } \phi=\frac{1-2 \exp \left(\sigma^{2}\right)}{1-\exp \left(\sigma^{2}\right)} .
\end{aligned}
$$

The proposal density is used in the Metropolis/Hasting algorithm within a new block, e.g. following the step 3 in the MCMC procedure for the jump-diffusion processes. This block updates all the variances $V_{i}, i=1, \ldots, T$. For $V_{1}$ and $V_{\mathrm{T}}$ the formula (19) needs to be modified slightly since $V_{0}$ and $V_{\mathrm{T}}$ are not known. The diffusion volatility $\sigma$ is obviously replaced by the square root of the latest estimate of the variance $V_{i}$ and we also need to add a new MCMC step for the AR(1) coefficients $\alpha, \beta$ and $\gamma$ (for example following the step that updates $\mathbf{V}$ ). The coefficients $\alpha, \beta$ can be sampled with a bivariate normal distribution, and $\gamma$ with the inverse gamma distribution. The extended MCMC algorithm is in detail described as follows:

3 Lognormal probability density fiction with parameters $\mu$ and $\sigma$ is given by

$L N(x ; \mu, \sigma)=\frac{1}{x \sigma \sqrt{2 \pi}} \exp \left(-0.5(\log x-\mu)^{2} / \sigma^{2}\right)$. It is useful to note that the mean of $\mathrm{x}$ is $\exp \left(\mu+\sigma^{2} / 2\right)$ and the variance is $\left(\exp \left(\sigma^{2}\right)-1\right) \exp \left(2 \mu+\sigma^{2}\right)$. 
1. Sample reasonable initial values $\mu^{(0)}, \lambda^{(0)}, \mu_{J}^{(0)}, \sigma_{J}{ }^{(0)}, \alpha^{(0)}, \beta^{(0)}, \gamma^{(0)}, \mathbf{V}^{(0)}, \mathbf{Z}^{(0)}, \mathbf{J}^{(0)}$

2. For $i=1, \ldots, T$ sample $Z_{i}^{(g)} \propto \varphi\left(Z ; \mu_{J}^{(g-1)}, \sigma_{J}^{(g-1)}\right)$ if $J_{i}^{(g-1)}=0$, and

$$
Z_{i}^{(g)} \propto \varphi\left(r_{i} ; \mu^{(g-1)}+Z, \sqrt{V_{i}^{(g-1)}}\right) \varphi\left(Z ; \mu_{J}^{(g-1)}, \sigma_{J}^{(g-1)}\right) \text { if } J_{i}^{(g-1)}=1 .
$$

3. For $i=1, \ldots, T$ sample $J_{i}^{(g)} \in\{0,1\}, \operatorname{Pr}[J=1]=p_{1} /\left(p_{0}+p_{1}\right)$, where

$$
\begin{aligned}
& p_{0}=\varphi\left(r_{i} ; \mu^{(g-1)}, \sqrt{V_{i}^{(g-1)}}\right)\left(1-\lambda^{(g-1)}\right), \\
& p_{1}=\varphi\left(r_{i} ; \mu^{(g-1)}+Z, \sqrt{V_{i}^{(g-1)}}\right) \lambda^{(g-1)}
\end{aligned}
$$

4. Sample new stochastic variances $V_{i}^{(g)}$ for $i=1, \ldots, T$ using Metrolis-Hastings (14) with the proposal density given by (20)

5. Sample new stochastic volatility autoregression coefficients $\alpha^{(g)}, \beta^{(g)}, \gamma^{(g)}$ from $h_{i}=\log V_{i}^{(g)}$ for $i=1, \ldots, T$ using the Bayesian linear regression model (Lynch, 2007):

$$
\begin{aligned}
& \hat{\boldsymbol{\beta}}=\left(\mathbf{X}^{\prime} \mathbf{X}\right)^{-1} \mathbf{X y}, \hat{\mathbf{e}}=\mathbf{y}-\mathbf{X} \hat{\boldsymbol{\beta}}, \text { where } \mathbf{X}=\left(\begin{array}{l}
1 \ldots .1 \\
h_{1} \ldots h_{T-1}
\end{array}\right)^{\prime}, \mathbf{y}=\left(h_{2} \ldots h_{T}\right)^{\prime}, \\
& \left(\gamma^{(g)}\right)^{2} \propto I G\left(\frac{n-2}{2}, \frac{\hat{\mathbf{e}}^{\prime} \hat{\mathbf{e}}}{2}\right), \\
& \left(\alpha^{(g)}, \beta^{(g)}\right)^{\prime} \propto \varphi\left((\alpha, \beta)^{\prime} ; \hat{\boldsymbol{\beta}},\left(\gamma^{(g)}\right)^{2}\left(\mathbf{X}^{\prime} \mathbf{X}\right)^{-1}\right) .
\end{aligned}
$$

6. Sample $\mu^{(g)}$ based on the normally distributed time series $r_{i}-Z_{i}^{\left({ }^{(g)}\right.} J_{i}^{(g)}$ with variances $V_{i}^{(g)}$, i.e.

$$
p\left(\mu^{(g)} \mid \mathbf{r}, \mathbf{Z}^{(g)}, \mathbf{J}^{(g)}, \mathbf{V}^{(g)}\right) \propto \varphi\left(\mu ; \sum_{i=1}^{T} \frac{r_{i}-J_{i}^{(g)} Z_{i}^{(g)}}{V_{i}^{(g)}} / \sum_{i=1}^{T} \frac{1}{V_{i}^{(g)}}, 1 / \sum_{i=1}^{T} \frac{1}{V_{i}^{(g)}}\right) .
$$

7. Sample $\lambda^{(0)}$ based on Bernoulli distributed $J_{i}^{(g)}$ binary time series according to (13).

8. Sample $\mu_{j}^{(g)}, \sigma_{j}^{(g)}$ based on the normally distributed time series $Z_{i}^{(g)}$ according to (9) and (10).

\subsection{Bivariate Jump-diffusion Stochastic Volatility Model}

Our ultimate goal is to study relationship between returns, jumps, and volatilities of two related financial series. First, we may estimate independently the parameters and latent variables $\mathbf{J}_{1}, \mathbf{Z}_{1}, \mathbf{V}_{1}$ and $\mathbf{J}_{2}, \mathbf{Z}_{2}, \mathbf{V}_{2}$ of (16) for two given series of returns $\mathbf{r}_{1}$ and $\mathbf{r}_{2}$. Since we also get estimates of the latent variables, i.e. jump times, jump sizes, and variances, we may inspect their relationship. For example we may analyze the overlap of probable jump times of the two processes, i.e. of the sets $\left\{i \mid P\left[J_{1, i}=1\right]>0.5\right\}$ and $\left\{i \mid P\left[J_{2, i}=1\right]>0.5\right\}$. Similarly the relationship between mean volatilities can be analyzed. Since $h_{1, i}=\log V_{1, i}$ is specified as a normal variable by the model, the mean volatility should be expressed by $\bar{\sigma}_{1, i}=\exp \left(\bar{h}_{1, i} / 2\right)$ where $\bar{h}_{1, i}$ is an MCMC estimation of the mean of $V_{1, i}$. The correlation between the series $\left\langle\bar{\sigma}_{1, i} ; i=1, \ldots, T\right\rangle$ and $\left\langle\bar{\sigma}_{2, i} ; i=1, \ldots, T\right\rangle$ (residuals or levels) indicates what the correlation between the stochastic volatilities might be. 
However, to give a consistent answer we need to specify a full bivariate stochastic volatility jump diffusion model:

$$
\begin{aligned}
& r_{1, i}=\mu_{1}+\sqrt{V_{1, i}} \epsilon_{1, i}+Z_{1, i} J_{1, i} \\
& \log V_{1, i}=\alpha_{1}+\beta_{11} \log V_{1, i-1}+\beta_{12} \log V_{2, i-1}+\gamma_{1} \epsilon_{1, i}^{V} \\
& r_{2, i}=\mu_{2}+\sqrt{V_{2, i}} \epsilon_{2, i}+Z_{2, i} J_{2, i} \\
& \log V_{2, i}=\alpha_{2}+\beta_{21} \log V_{1, i-1}+\beta_{22} \log V_{2, i-1}+\gamma_{2} \epsilon_{2, i}^{V} \\
& \left(\begin{array}{c}
\epsilon_{1, i} \\
\epsilon_{2, i}
\end{array}\right) \sim N\left(0,\left(\begin{array}{ll}
1 & \rho \\
\rho & 1
\end{array}\right)\right),\left(\begin{array}{c}
\epsilon_{1, i}^{V} \\
\epsilon_{2, i}^{V}
\end{array}\right) \sim N\left(0,\left(\begin{array}{cc}
1 & \rho_{V} \\
\rho_{V} & 1
\end{array}\right)\right) \\
& \left(\begin{array}{l}
Z_{1, i} \\
Z_{2, i}
\end{array}\right) \sim N\left(\left(\begin{array}{l}
\mu_{J 1} \\
\mu_{J 2}
\end{array}\right),\left(\begin{array}{cc}
\sigma_{J 1}^{2} & \rho_{Z} \sigma_{J 1} \sigma_{J 2} \\
\rho_{Z} \sigma_{J 1} \sigma_{J 2} & \sigma_{J 2}^{2}
\end{array}\right)\right) \\
& J_{1, i} \sim \operatorname{Bern}\left(\lambda_{1}\right), J_{2, i} \sim \operatorname{Bern}\left(\lambda_{2}\right), \operatorname{corr}\left(J_{1, i} J_{2, i}\right)=\rho_{J}
\end{aligned}
$$

The model does not take into account a possible correlation between the diffusion and log-variance residuals, but it incorporates possible Granger causality between the two $\log$-variance processes. For example $\beta_{12}>0$ if then high level of $\log V_{2}$ (Granger) causes $\log V_{1}$ to become larger. Our preliminary analysis of the data we intend to study indeed indicates that the volatility residuals $\epsilon_{1, i}^{V}$ and $\epsilon_{2, i}^{V}$ are not correlated, but $\log V_{1 i}$ and $\log V_{2 i}$ are correlated. We also compare the results of the stochastic volatility jump-diffusion model against the restricted bivariate jump-diffusion model with constant volatilities, inspecting in particular the jump probabilities and the jump correlations estimated with and without stochastic volatilities.

\section{Correlated jumps}

In order to incorporate correlated jumps and jump sizes into the MCMC algorithm given in Section 2.3 we need to modify the steps 2 and 3 given as follows:

Sample the new jump sizes (omitting the upper indices $g$ and $g-1$ )

where

$$
\begin{aligned}
& p\left(Z_{i} \mid r_{i}, J_{i}, \Theta\right) \propto p\left(r_{i} \mid Z_{i}, J_{i}, \Theta\right) p\left(Z_{i} \mid \Theta\right) \\
& \propto \exp \left(-0.5\left(y_{i}{ }^{\prime} \Sigma_{i} y_{i}+\left(Z_{i}-\mu_{Z}\right)^{\prime} \Sigma_{J}\left(Z_{i}-\mu_{Z}\right)\right)\right) \\
& \propto \exp \left(-0.5\left(Z_{i}-m_{i}\right)^{\prime} V_{i}^{-1}\left(Z_{i}-m_{i}\right)\right)
\end{aligned}
$$

$$
\begin{gathered}
r_{i}=\left(\begin{array}{l}
r_{1, i} \\
r_{2, i}
\end{array}\right), \mu=\left(\begin{array}{l}
\mu_{1} \\
\mu_{2}
\end{array}\right), Z_{i}=\left(\begin{array}{l}
Z_{1, i} \\
Z_{2, i}
\end{array}\right), J_{i}=\left(\begin{array}{cc}
J_{1, i} & 0 \\
0 & J_{2, i}
\end{array}\right), y_{i}=r_{i}-\mu-J_{i} Z_{i}, \\
\Sigma_{i}=\left(\begin{array}{cc}
V_{1, i} & \rho \sqrt{V_{1, i} V_{2, i}} \\
\rho \sqrt{V_{1, i} V_{2, i}} & V_{2, i}
\end{array}\right), \Sigma_{J}=\left(\begin{array}{cc}
\sigma_{J 1}^{2} & \rho_{Z} \sigma_{J 1} \sigma_{J 2} \\
\rho_{Z} \sigma_{J 1} \sigma_{J 2} & \sigma_{J 2}^{2}
\end{array}\right)
\end{gathered}
$$




$$
V_{i}=\left(J_{i} \Sigma_{i}^{-1} J_{i}+\Sigma_{J}^{-1}\right)^{-1}, m_{i}=V_{i}\left(J_{i} \Sigma_{i}^{-1}\left(r_{i}-\mu_{i}\right)+\Sigma_{J}^{-1}\right)
$$

Next, we need to resample the correlated jump times. Since by definition

$$
\begin{gathered}
\rho_{J}=\frac{p_{11}-\lambda_{1} \lambda_{2}}{\sqrt{\lambda_{1} \lambda_{2}\left(1-\lambda_{1}\right)\left(1-\lambda_{2}\right)}} \text { where } p_{11}=\operatorname{Pr}\left[J_{1, i}=1 \& J_{2, i}=1\right] \text { we have } \\
\operatorname{Pr}\left[J_{1, i}=1 \mid J_{2, i}=1\right]=\lambda_{1}+\rho_{J} \frac{\sqrt{\lambda_{1}\left(1-\lambda_{1}\right)\left(1-\lambda_{2}\right)}}{\sqrt{\lambda_{2}}} \text { and } \\
\operatorname{Pr}\left[J_{1, i}=1 \mid J_{2, i}=0\right]=\lambda_{1}-\rho_{J} \frac{\sqrt{\lambda_{1} \lambda_{2}\left(1-\lambda_{1}\right)}}{\sqrt{1-\lambda_{2}}} .
\end{gathered}
$$

Similarly to (21) (again omitting the upper indices $g$ and $g-1$ ) we set

$$
\begin{aligned}
& p_{0}=\varphi\left(r_{i} ;\left\langle\mu_{1}, \mu_{2}-Z_{2, i} J_{2, i}\right\rangle^{\prime}, \Sigma_{i}\right) \operatorname{Pr}\left[J_{1, i}=0 \mid J_{2, i}\right], \\
& p_{1}=\varphi\left(r_{i} ;\left\langle\mu_{1}-Z_{1, i}, \mu_{2}-Z_{2, i} J_{2, i}\right\rangle^{\prime}, \Sigma_{i}\right) \operatorname{Pr}\left[J_{1, i}=1 \mid J_{2, i}\right],
\end{aligned}
$$

and sample $J_{1, i} \in\{0,1\}, \operatorname{Pr}\left[J_{1, i}=1\right]=p_{1} /\left(p_{0}+p_{1}\right)$.

Finally, we need to add two additional steps re-sampling the correlations $\rho_{J}$ and $\rho_{J}$. The jump size correlation can be in fact sampled in one step with jump size volatilities $\sum_{J}$ using the inverse Wishart distribution (12) as the series $Z_{i}$ is assumed to be bivariate normal. Regarding $\rho_{J}$ we use the random walk Metropolis-Hastings step and the relationship

$$
\begin{gathered}
p\left(\rho_{J} \mid \mathbf{J}_{1}, \mathbf{J}_{2}, \lambda_{1}, \lambda_{2}\right) \propto L\left(\mathbf{J}_{1}, \mathbf{J}_{2} \mid \rho_{J}, \lambda_{1}, \lambda_{2}\right)=\prod_{i=1}^{T} p\left(J_{1, i}, J_{2, i}\right), \text { where } \\
p(1,1)=\lambda_{1} \lambda_{2}+\rho_{J} \sqrt{\lambda_{1} \lambda_{2}\left(1-\lambda_{1}\right)\left(1-\lambda_{2}\right)}, p(1,0)=\lambda_{1}-p(1,1), \\
p(0,1)=\lambda_{2}-p(1,1), p(0,0)=1-\lambda_{1}-p(0,1) .
\end{gathered}
$$

Regarding the diffusion residuals correlation coefficient $\rho$ it can be easily re-sampled using the random walk Metropolis-Hastings:

$$
p\left(\rho \mid \mathbf{r}_{1}, \mathbf{J}_{1}, \mathbf{Z}_{1}, \mathbf{V}_{\mathbf{1}}, \mathbf{r}_{\mathbf{2}}, \mathbf{J}_{\mathbf{2}}, \mathbf{Z}_{\mathbf{2}}, \mathbf{V}_{2}, \Theta\right) \propto \prod_{i=1}^{T} \varphi\left(r_{i} ; \mu, \Sigma_{i}\right)
$$

with the bivariate normal density function and the notation (24) .

\section{Correlated stochastic volatilities}

Correlation between stochastic volatilities can be captured by correlated residuals and by correlated levels of the log volatilities expressed in the VAR(1) model (23).

In order to simplify the notation set $h_{k, i}=\log V_{k, i},(k=1,2 ; i=1, \ldots, T)$. Re-sampling 
the variance $V_{1, i}$ conditional on the other latent variables and parameters MCMC needs to take into account its relationship to $r_{i}=\left\langle r_{1, i}, r_{2, i}\right\rangle$ and to $V_{1, i-1}, V_{2, i-1}$ given by the VAR equation for $h_{1, i}$, but also to $V_{1, i+1}$ and $V_{2, i+1}$ given by the equations for $h_{1, i+1}$ and $h_{2, i+2}$. Applying the Bayes theorem:

$$
\begin{aligned}
& p\left(V_{1, i} \mid \mathbf{V}_{1,(-i)}, \mathbf{V}_{2}, \Theta, \mathbf{r}_{1}, \mathbf{Z}_{1}, \mathbf{J}_{1}, \mathbf{r}_{2}, \mathbf{Z}_{2}, \mathbf{J}_{2}\right) \\
& \propto p\left(r_{i} \mid V_{i}, \Theta, Z_{i}, J_{i}\right) p\left(V_{1, i} \mid V_{1, i-1}, V_{2, i-1}, \Theta\right) p\left(V_{1, i+1} \mid V_{1, i}, V_{2, i}, \Theta\right) p\left(V_{2, i+1} \mid V_{1, i}, V_{2, i}, \Theta\right) .
\end{aligned}
$$

In order to set up a manageable proposal density let us assume that $r_{1, i}, r_{2, i}$ are uncorrelated and take into account only the likelihood of the return $r_{1, i}$ given the other parameters. True proportional density used the Metropolis- Hastings step will be still given by (25). After this modification, the first probability distribution on the right hand side is again inverse gamma in $V_{1, i}$, while the remaining densities are lognormal. The product of the three lognormal densities is lognormal with the corresponding normal distribution mean and variance:

$$
\begin{aligned}
\mu_{i} & =\frac{\gamma_{2}^{2}\left(\alpha_{1}+\beta_{11} h_{1, i-1}+\beta_{12} h_{2, i-1}\right)+\gamma_{2}^{2} \beta_{11}\left(h_{1, i+1}-\alpha_{1}-\beta_{12} h_{2, i}\right)+\gamma_{1}^{2} \beta_{21}\left(h_{2, i+1}-\alpha_{2}-\beta_{22} h_{2, i}\right)}{\gamma_{2}^{2}\left(1+\beta_{11}^{2}\right)+\gamma_{1}^{2} \beta_{21}^{2}}, \\
\sigma^{2} & =\frac{\gamma_{1}^{2} \gamma_{2}^{2}}{\gamma_{2}^{2}\left(1+\beta_{11}^{2}\right)+\gamma_{1}^{2} \beta_{21}^{2}} .
\end{aligned}
$$

The Metropolis-Hastings proposal density for updated $V_{1, i}$ is then given by (20). The second process varianceis $V_{2, i}$ resampled analogously.

Finally, given $\mathbf{V}_{1}$ and $\mathbf{V}_{2}$ (omitting the upper index $g$ ) we need to update the coefficients $\alpha_{1}, \beta_{11}, \beta_{12}, \gamma_{1}$, and $\alpha_{2}, \beta_{21}, \beta_{22}, \gamma_{2}$. We shall use the Bayesian linear regression model as in the univariate case:

$$
\begin{aligned}
& \hat{\boldsymbol{\beta}}=\left(\mathbf{X}^{\prime} \mathbf{X}\right)^{-1} \mathbf{X} \mathbf{y}, \hat{\mathbf{e}}=\mathbf{y}-\mathbf{X} \hat{\boldsymbol{\beta}}, \text { where } \mathbf{X}=\left(\begin{array}{l}
1 \ldots .1 \\
h_{1,1} \ldots h_{1, T-1} \\
h_{2,1} \ldots h_{2, T-1}
\end{array}\right)^{\prime}, \mathbf{y}=\left(h_{1,2} \ldots h_{1, T}\right)^{\prime}, \\
& \gamma_{1}^{2} \propto I G\left(\frac{n-3}{2}, \frac{\hat{\mathbf{e}}^{\prime} \hat{\mathbf{e}}}{2}\right), \\
& \left(\alpha_{1}, \beta_{11}, \beta_{12}\right)^{\prime} \propto \varphi\left(\left(\alpha_{1}, \beta_{11}, \beta_{12}\right)^{\prime} ; \hat{\boldsymbol{\beta}}, \gamma_{1}^{2}\left(\mathbf{X}^{\prime} \mathbf{X}\right)^{-1}\right) .
\end{aligned}
$$

Similarly we proceed for $\alpha_{2}, \beta_{21}, \beta_{22}, \gamma_{2}$.

\subsection{Model Comparison and Value at Risk Estimations}

In order to compare the different models we will use the deviance information criterion (DIC) of Spielgelhalter et al. (2002). It is shown to generalize the Akaike information criterion (AIC) that is not appropriate to compare stochastic volatility or jump-diffusion 
models (Yu, Meyer, 2006). In order to calculate AIC one needs to specify the number of free parameters. In case of the stochastic volatility and jump-diffusion models the number of additional latent variables estimated has an order of $T$. On the other hand, since the variables are not independent, it is not clear what should be the number of free variables. Likewise AIC, DIC has two components, a term that measures goodness-of-fit and a penalty term for increasing model complexity:

$$
D I C=\bar{D}+p_{D} .
$$

The first term is defined as the posterior mean of the deviance $D(\Theta)=-2 \log L($ data $\mid \Theta)$, i.e.

$$
\bar{D}=E_{\Theta \mid \text { data }}[D(\Theta)]
$$

Here $\Theta$ includes all the model parameters and estimated state variables. Since the likelihood function $L($ data $\mid \Theta)$ is known the measure is estimated just averaging the likelihood over MCMC sampled estimates of $\Theta^{(g)}$.

The second term measuring the effective number of parameters is defined as the difference between the posterior mean of the deviance and the deviance evaluated at the estimated parameters $\hat{\Theta}$, i.e.

$$
p_{D}=\bar{D}-D(\hat{\Theta}) .
$$

The parameters are, in our case, estimated as means of the sampled values discarding an initial period of the MCMC sampling. The stochastic volatilities are estimated as $\hat{\sigma}_{k, i}=\exp \left(\bar{h}_{k, i} / 2\right)$ where $\bar{h}_{k, i}$ is the mean of sampled values. In case of jump occurrence we set $\hat{J}_{k, i}=1$ if and only if the mean of sampled jump indicators is larger than 0.5 , i.e. if the probability of jump occurrence is estimated to be larger than $50 \%$. The jump size is again estimated as the sample mean of sampled jumps.

Other classical Bayesian goodness-of-fit measures to be mentioned are the Bayes factors or marginal likelihood. We follow Yu and Meyer (2006) who recommend the DIC measure shown to have a consistent performance with respect to the two standard measures, and is relatively easy to compute.

\section{Value at Risk (VaR)}

Finally, we intend to illustrate the difference between the estimated bivariate models calculating $\mathrm{VaR}$ for different time horizons and confidence levels. If the true data were generated by a simple process, e.g. the pure diffusion one, then the estimations yielded by more general models (including jumps and/or stochastic volatilities) but calibrated on the same dataset should be similar. However, if the data contain "true" jumps and/or stochastic volatility, then the VaR estimations will probably significantly differ, and the choice of model becomes important.

Formally, Value at Risk, $\operatorname{VaR}(N, 1-\alpha)$ is defined as $-q_{\alpha}[X]$ where $q_{\alpha}[X]$ denotes the $\alpha-$ quantile of the random variable $X$ modelling the portfolio market profit/loss, $N$ (business) 
days to the future. The variable $X$ can be also defined as the portfolio return relative to the initial investment.

We will consider a simple foreign stock investment where the domestic currency value of the portfolio is $V^{D C}=S \cdot V^{F C}$, is the exchange rate measuring foreign currency $F C$ in terms of domestic currency $D C$, and $V^{F C}$ is the foreign currency stock value. The domestic currency log-return over a time horizon can be expressed as the sum $r^{D C}=r^{S+} r^{F C}$ of the exchange rate log-return and the foreign currency stock log-return. Thus, in order to get the distribution and quantiles of $r^{D C}$ we need to model the joint distribution of $r^{S}$ and $r^{F C}$.

The simplest and widely used (parametric normal VaR) method calculates the variances and the covariance of $r^{S}$ and $r^{F C}$, combines them to get the standard deviation of $r^{D C}=r^{S+} r^{F C}$, and multiplies it with a standard normal distribution $1-\alpha$ quantile to get an estimate of $\operatorname{VaR}(1,1-\alpha)$. To get $N$ day estimate, the value is scaled by $\sqrt{ } N$. We will use the estimation with an EWMA (exponentially moving weighted average) covariance matrix as a benchmark value.

Given a specific stochastic model and its parameters we may simulate the returns $r_{T+1}, \ldots, r_{T+1}$ one to $N$ days ahead, and so the compounded $N$-days log-return $r=\sum_{i=1}^{N} r_{T+i}$. Note that in case of the stochastic volatility model (16) the initial values must also include an estimate of the last variance $V_{T}$. The bivariate model jump-diffusion model with stochastic volatility model (23) allows us to simulate the joint distribution of $r^{S}$ and $r^{F C}$ over $N$ days horizon.

The Bayesian estimations in fact yields an empirical distribution of the parameters approximated by a sequence of MCMC simulated values $\Theta_{1}, \ldots, \Theta_{K}$ (discarding an initial sampling period). The Value at Risk estimated as above is then conditional on the estimate $\hat{\Theta}$, i.e. in fact we get $\widehat{\operatorname{VaR}}(N, 1-\alpha \mid \hat{\Theta})$. Since the parameters are uncertain and modified parameters might influence significantly the VaR measure we should rather calculate a VaR estimate incorporating the parameter uncertainty. This can be easily done in the Bayesian framework sampling firstly $\Theta_{i}$ from $\Theta_{1}, \ldots, \Theta_{K}$ and then simulating the returns given the parameter vector $\Theta_{i}$. The distribution of simulated returns can be used to obtain the Bayesian Value at Risk denoted as $\widehat{\mathrm{VaR}}(N, 1-\alpha \mid$ data $)$, i.e. VaR conditional on the data used to estimate the parameters rather than on a specific vector of point estimates.

\section{Empirical Study}

The main goal of this section is to apply the bivariate jump-diffusion model stochastic volatility model, its submodels (bivariate diffusion, jump-diffusion, and stochastic volatility), and the proposed estimation method to the FX and stock market time series returns. However, before doing so we are going to test the estimation procedures on artificially generated data in order to demonstrate that the model is able to identify or reject jumps and stochastic volatility parameters. The MCMC algorithm and all calculations have been implemented in Matlab. 


\subsection{An Empirical Test}

We are going to sample $T=2000$ returns according to the bivariate jump-diffusion stochastic volatility model with several sets of the model parameters (and starting with initial $\log$-variances $\left.\log V_{1,0}=\log V_{2,0}-7\right)$. The proposed MCMC estimation method is then applied to the generated data and estimated parameters are compared with the original parameters that were used to generate the data. The "true" parameters are expected to lie with the Bayesian confidence intervals.

The upper part of Table 1 shows parameters of the first artificially generated bivariate process. There are no jumps, there is no correlation between the diffusion residuals, and no correlation between the variances. The lower part of the table shows the MCMC estimates with standard deviations in parenthesis. The MCMC procedure has been run 3000 times and the first 1000 estimations have been discarded. Figure 1 shows, for example, relatively fast convergence of the first stochastic volatility equation coefficients $\beta_{11}$ and $\beta_{12}$.

Table 1.

Generated Bivariate Stochastic Volatility Process (upper table) and the MCMC Estimated Parameters

\begin{tabular}{|c|c|c|c|c|c|c|c|}
\hline \multicolumn{7}{|c|}{ Generated jump-diffusion process 1 with stochastic volatility } \\
\hline$\mu_{1}$ & $\lambda_{1}$ & $\mu_{1, J}$ & $\sigma_{1, J}$ & $\alpha_{1}$ & $\beta_{11}$ & $\beta_{12}$ & $\gamma_{1}$ \\
\hline 0.001 & 0 & - & - & -0.14 & 0.98 & 0 & 0.13 \\
\hline \multicolumn{7}{|c|}{ Generated jump-diffusion process 2 with stochastic volatility } \\
\hline$\mu_{2}$ & $\lambda_{2}$ & $\mu_{2, J}$ & $\sigma_{2,\lrcorner}$ & $\alpha_{2}$ & $\beta_{21}$ & $\beta_{22}$ & $\gamma_{2}$ \\
\hline 0.003 & 0 & - & - & -0.28 & 0 & 0.96 & 0.16 \\
\hline \multicolumn{7}{|c|}{ Correlations } \\
\hline
\end{tabular}

\begin{tabular}{|c|c|c|c|c|c|c|c|}
\hline \multicolumn{8}{|c|}{ Estimated jump-diffusion process 1 with stochastic volatility } \\
\hline$\mu_{1}$ & $\lambda_{1}$ & $\mu_{1, J}$ & $\sigma_{1, J}$ & $\alpha_{1}$ & $\beta_{11}$ & $\beta_{12}$ & $\gamma_{1}$ \\
\hline $\begin{array}{c}0.0022 \\
(4.477 \mathrm{e}-004)\end{array}$ & $\begin{array}{c}0.0026 \\
(0.0050)\end{array}$ & $\begin{array}{l}-0.0041 \\
(0.0421)\end{array}$ & $\begin{array}{c}0.0893 \\
(0.0108)\end{array}$ & $\begin{array}{l}-0.1337 \\
(0.0595)\end{array}$ & $\begin{array}{c}0.9794 \\
(0.0069)\end{array}$ & $\begin{array}{c}0.0020 \\
(0.0066)\end{array}$ & $\begin{array}{c}0.1233 \\
(0.0161)\end{array}$ \\
\hline \multicolumn{8}{|c|}{ Estimated jump-diffusion process 2 with stochastic volatility } \\
\hline$\mu_{2}$ & $\lambda_{2}$ & $\mu_{2, J}$ & $\sigma_{2, J}$ & $\alpha_{2}$ & $\beta_{21}$ & $\beta_{22}$ & $\gamma_{2}$ \\
\hline $\begin{array}{c}0.0023 \\
(4.606 \mathrm{e}-004)\end{array}$ & $\begin{array}{c}0.0045 \\
(0.0025)\end{array}$ & $\begin{array}{c}0.0304 \\
(0.0224)\end{array}$ & $\begin{array}{c}0.1021 \\
(0.0242)\end{array}$ & $\begin{array}{l}-0.1825 \\
(0.0603)\end{array}$ & $\begin{array}{c}0.0011 \\
(0.0059)\end{array}$ & $\begin{array}{c}0.9730 \\
(0.0074)\end{array}$ & $\begin{array}{c}0.1332 \\
(0.0138)\end{array}$ \\
\hline \multicolumn{8}{|c|}{ Estimated correlations } \\
\hline \multicolumn{2}{|c|}{$\rho$} & \multicolumn{3}{|c|}{$\rho_{\jmath}$} & \multicolumn{3}{|c|}{$\rho_{z}$} \\
\hline \multicolumn{2}{|c|}{$-0.0056(0.0243)$} & \multicolumn{3}{|c|}{$0.4275(0.1920)$} & \multicolumn{3}{|c|}{$0.0764(0.5542)$} \\
\hline
\end{tabular}


The estimated stochastic volatility parameters are consistent with the "true" parameters, indeed the estimated coefficients $\beta_{12}$ and $\beta_{21}$ are not significantly different from zero and so Granger causality would not be detected. The estimated means $\mu_{1}$ and $\mu_{2}$ seem to differ from the "true" values significantly, but we have to take into account the error caused by the data generation process itself possibly correlated to a relatively large volatility. The mean of the first generated return series turns out to be 0.0023 and of the second 0.0019 in line with the estimations. The zero jump intensities fall within the $95 \%$ confidence intervals around the estimated values. In fact no jumps with probability larger than $0.5 \%$ are identified by the sampled jump probabilities (averaging the jump indicator $J_{k, i}$ ) - see Figure 2.

Figure 1

Convergence of the Coefficients $\beta_{11}$ and $\beta_{12}$
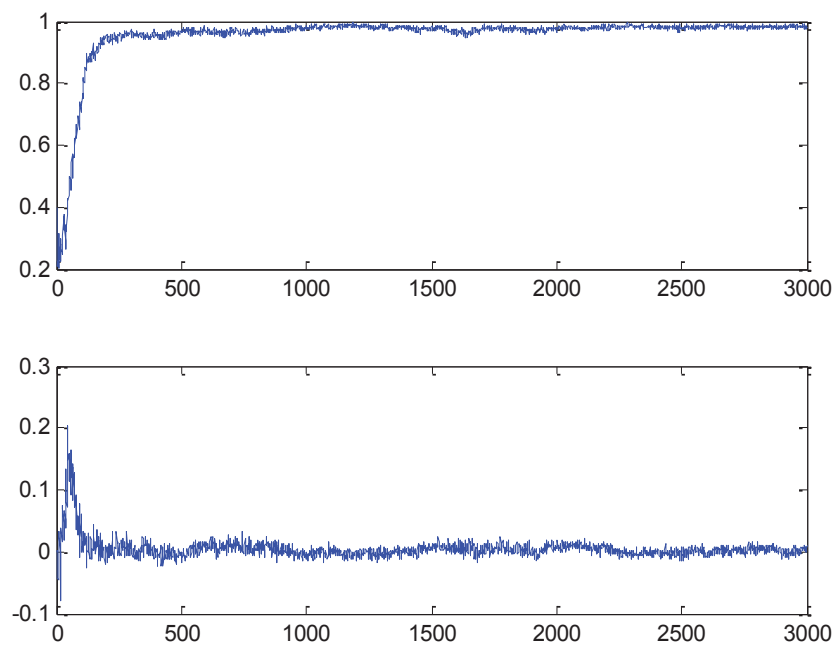

Figure 2

\section{Sampled Jump Probabilities}
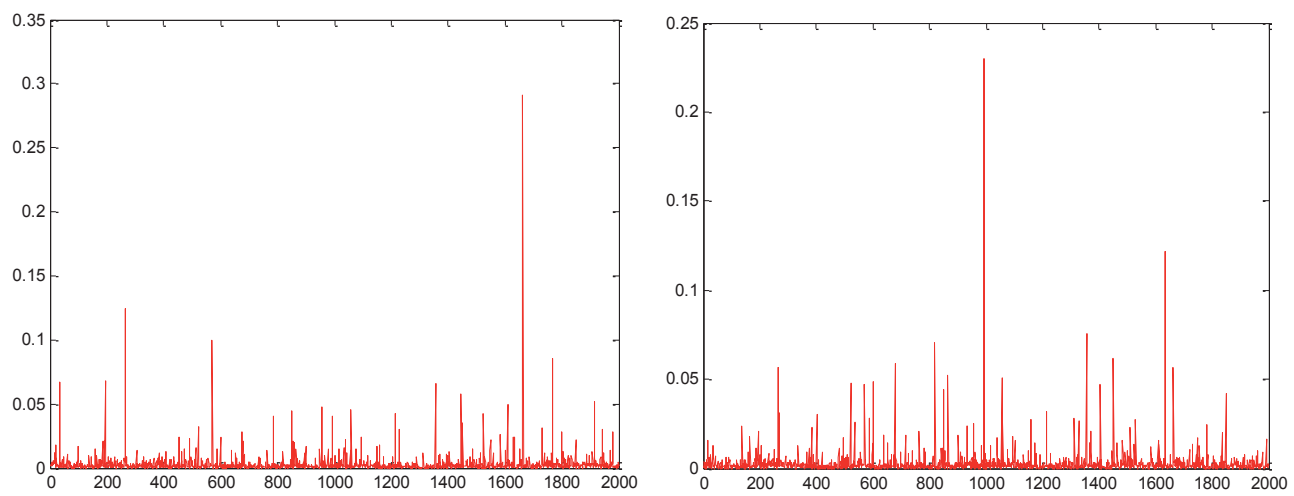
Table 2 shows the generating and estimated parameters of a process with nonzero jump intensities, with a positive jump occurrence correlation, but still with zero variance correlation. The estimates are again more-or-less consistent with the "true" parameters. Figure 3 tries to compare the simulated jumps and their absolute sizes with the estimated jump probabilities. The point is that if a simulated jump size is too small, then the jump can be ex post hardly identified and the estimated jump probability is low. This may explain the difference between the true jump probability $\lambda_{1}=0.0013$ and the estimated value $0.0037(0.0127)$. The precision of the jump intensity estimates should improve with a larger number of observations.

Table 2

Generated Bivariate Jump-diffusion Stochastic Volatility Process (upper table) and the MCMC Estimated Parameters

\begin{tabular}{|c|c|c|c|c|c|c|c|}
\hline \multicolumn{8}{|c|}{ Generated jump-diffusion process 1 with stochastic volatility } \\
\hline$\mu_{1}$ & $\lambda_{1}$ & $\mu_{1, J}$ & $\sigma_{1, J}$ & $\alpha_{1}$ & $\beta_{11}$ & $\beta_{12}$ & $V_{1}$ \\
\hline 0.003 & 0.0130 & 0.03 & 0.15 & -0.14 & 0.98 & 0 & 0.13 \\
\hline \multicolumn{8}{|c|}{ Generated jump-diffusion process 2 with stochastic volatility } \\
\hline$\mu_{2}$ & $\lambda_{2}$ & $\mu_{2, J}$ & $\sigma_{2, J}$ & $\alpha_{2}$ & $\beta_{21}$ & $\beta_{22}$ & $\gamma_{2}$ \\
\hline 0.001 & 0.0205 & -0.02 & 0.17 & -0.21 & 0 & 0.97 & 0.16 \\
\hline \multicolumn{8}{|c|}{ Correlations } \\
\hline \multicolumn{3}{|c|}{$\rho$} & \multicolumn{2}{|c|}{$\rho_{J}$} & \multicolumn{3}{|c|}{$\rho_{z}$} \\
\hline \multicolumn{3}{|c|}{0} & \multicolumn{2}{|c|}{0.2326} & \multicolumn{3}{|c|}{0} \\
\hline
\end{tabular}

\begin{tabular}{|c|c|c|c|c|c|c|c|}
\hline \multicolumn{8}{|c|}{ Estimated jump-diffusion process 1 with stochastic volatility } \\
\hline$\mu_{1}$ & $\lambda_{1}$ & $\mu_{1, J}$ & $\sigma_{1, J}$ & $\alpha_{1}$ & $\beta_{11}$ & $\beta_{12}$ & $V_{1}$ \\
\hline $\begin{array}{c}0.0026(4.876 \mathrm{e}- \\
004)\end{array}$ & $\begin{array}{c}0.0037 \\
(0.0127)\end{array}$ & $\begin{array}{c}0.0344 \\
(0.0392)\end{array}$ & $\begin{array}{c}0.1737 \\
(0.0168)\end{array}$ & $\begin{array}{l}-0.0941 \\
(0.0505)\end{array}$ & $\begin{array}{c}0.9836 \\
(0.0053)\end{array}$ & $\begin{array}{c}0.0029 \\
(0.0053)\end{array}$ & $\begin{array}{c}0.1206 \\
(0.0161)\end{array}$ \\
\hline \multicolumn{8}{|c|}{ Estimated jump-diffusion process 2 with stochastic volatility } \\
\hline$\mu_{2}$ & $\lambda_{2}$ & $\mu_{2, J}$ & $\sigma_{2, J}$ & $\alpha_{2}$ & $\beta_{21}$ & $\beta_{22}$ & $V_{2}$ \\
\hline $\begin{array}{l}4.7705 \mathrm{e}-004 \\
(4.574 \mathrm{e}-004)\end{array}$ & $\begin{array}{r}0.0290 \\
(0.0052)\end{array}$ & $\begin{array}{l}-0.0361 \\
(0.0223)\end{array}$ & $\begin{array}{c}0.1463 \\
(0.0144)\end{array}$ & $\begin{array}{l}-0.3125 \\
(0.0859)\end{array}$ & $\begin{array}{l}-0.0022 \\
(0.0080)\end{array}$ & $\begin{array}{c}0.9588 \\
(0.0101)\end{array}$ & $\begin{array}{c}0.1794 \\
(0.0197)\end{array}$ \\
\hline \multicolumn{8}{|c|}{ Estimated correlations } \\
\hline \multicolumn{2}{|l|}{$\rho$} & \multicolumn{3}{|c|}{$\rho_{J}$} & \multicolumn{3}{|c|}{$\rho_{z}$} \\
\hline \multicolumn{2}{|c|}{$-5.1738 \mathrm{e}-004(0.0243)$} & \multicolumn{3}{|c|}{$0.4026(0.1037)$} & \multicolumn{3}{|c|}{$0.0249(0.2574)$} \\
\hline
\end{tabular}


Figure 3

Bars below the $\mathrm{x}$-axis Show the Simulated Jumps and Their Absolute Size, Bars above the $\mathrm{x}$-axis Show the MCMC Estimated Jump Probabilities (left - process 1, right - process 2)
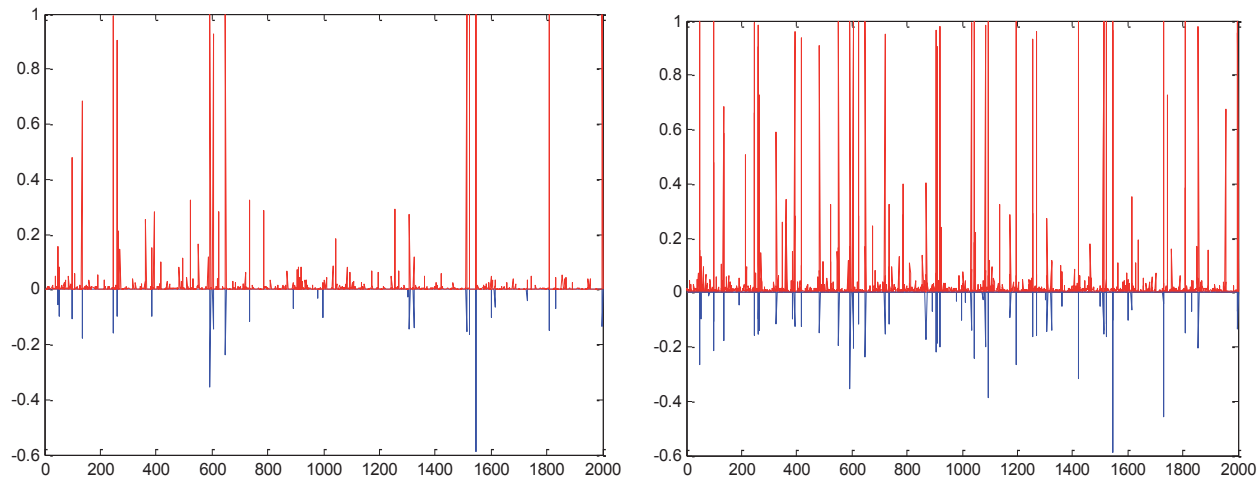

Finally Table 3 shows parameters and estimates of a bivariate jump-diffusion process with correlated stochastic volatilities. We have still set some parameters to zero, e.g. $\beta_{12}$ or $\rho$, in order to check that he estimation is able to confirm or reject significance of the individual parameters.

Table 3

Generated Bivariate Jump-diffusion Process (correlated) Stochastic Volatility Model (upper table) and the MCMC Estimated Parameters

\begin{tabular}{|c|c|c|c|c|c|c|c|}
\hline \multicolumn{8}{|c|}{ Generated jump-diffusion process 1 with stochastic volatility } \\
\hline$\mu_{1}$ & $\lambda_{1}$ & $\mu_{1, J}$ & $\sigma_{1, J}$ & $\alpha_{1}$ & $\beta_{11}$ & $\beta_{12}$ & $\gamma_{1}$ \\
\hline 0.003 & 0.0130 & 0.03 & 0.15 & -0.07 & 0.99 & 0 & 0.13 \\
\hline \multicolumn{8}{|c|}{ Generated jump-diffusion process 2 with stochastic volatility } \\
\hline$\mu_{2}$ & $\lambda_{2}$ & $\mu_{2, J}$ & $\sigma_{2, J}$ & $\alpha_{2}$ & $\beta_{21}$ & $\beta_{22}$ & $\gamma_{2}$ \\
\hline 0.001 & 0.0260 & -0.02 & 0.17 & -0.21 & 0.02 & 0.95 & 0.16 \\
\hline \multicolumn{8}{|c|}{ Correlations } \\
\hline \multicolumn{2}{|c|}{$\rho$} & \multicolumn{3}{|c|}{$\rho_{J}$} & \multicolumn{3}{|c|}{$\rho_{z}$} \\
\hline \multicolumn{2}{|c|}{0} & \multicolumn{3}{|c|}{0.3418} & \multicolumn{3}{|c|}{0} \\
\hline
\end{tabular}




\begin{tabular}{|c|c|c|c|c|c|c|c|}
\hline \multicolumn{7}{|c|}{ Estimated jump-diffusion process 1 with stochastic volatility } \\
\hline$\mu_{1}$ & $\lambda_{1}$ & $\mu_{1, J}$ & $\sigma_{1, J}$ & $\alpha_{1}$ & $\beta_{11}$ & $\beta_{12}$ & $\gamma_{1}$ \\
\hline 0.0018 & 0.0039 & 0.0256 & 0.1589 & -0.0931 & 0.9703 & 0.0157 & 0.1527 \\
$(5.261 \mathrm{e}-004)$ & $(0.0133)$ & $(0.0451)$ & $(0.0209)$ & $(0.0613)$ & $(0.0090)$ & $(0.0125)$ & $(0.0174)$ \\
\hline \multicolumn{7}{|c|}{ Estimated jump-diffusion process 2 with stochastic volatility } \\
\hline$\mu_{2}$ & $\lambda_{2}$ & $\mu_{2, J}$ & $\sigma_{2,\lrcorner}$ & $\alpha_{2}$ & $\beta_{21}$ & $\beta_{22}$ & $\gamma_{2}$ \\
\hline 0.0013 & 0.0288 & -0.0280 & 0.1800 & -0.2760 & 0.0280 & 0.9326 & 0.1676 \\
$(5.543 \mathrm{e}-004)$ & $(0.0051)$ & $(0.0275)$ & $(0.0199)$ & $(0.0931)$ & $(0.0099)$ & $(0.0186)$ & $(0.0254)$ \\
\hline \multicolumn{7}{|c|}{ Estimated correlations } \\
\hline \multicolumn{7}{|c|}{$0.3621(0.1264)$} & $\rho_{J}$ \\
\hline 0
\end{tabular}

During implementation of the models many empirical tests have been performed. We have shown only a few to illustrate a good performance of the proposed estimation algorithm.

\subsection{FX and Stock Market Data and Empirical Results}

Our data set consists of CZK/EUR exchange rates and the Czech PX stock index values from September 1, 2004 to February 2, 2011 (Figure 4). Note that the CZK/EUR is quoted as the inverse of the normal EUR/CZK exchange rate, i.e. as a direct quotation of CZK in terms of EUR from the perspective of an EUR investor. The period of strong decline of the stock index and depreciation of $\mathrm{CZK} / \mathrm{EUR}$ corresponds obviously to the financial crisis in 2007-2009.

Figure 4

CZK/EUR Exchange Rate and PX Stock Index Value September 1, 2004 to February 2, 2011

CZK/EUR

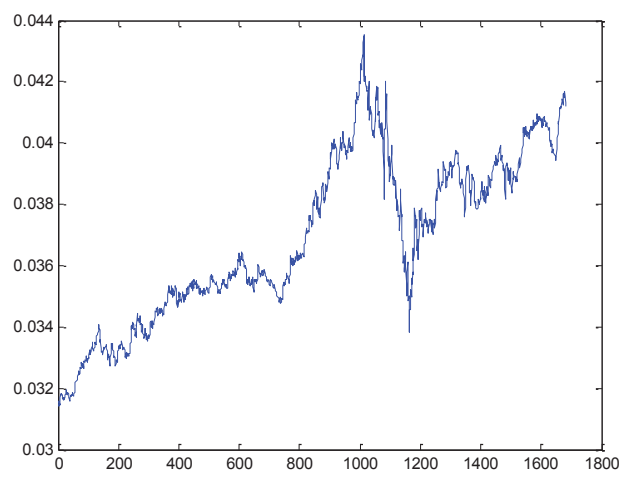

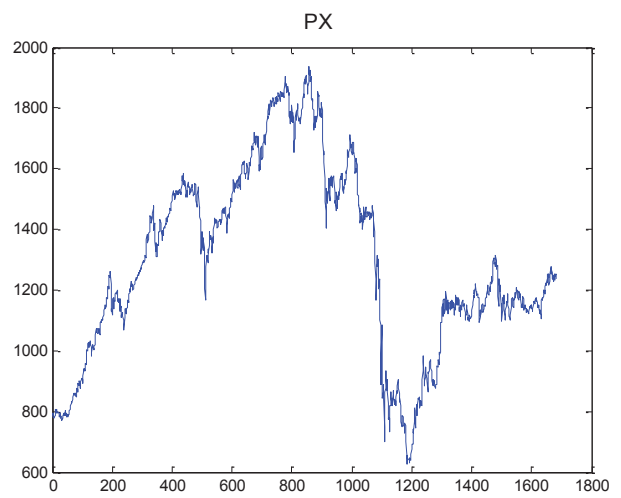


Time series of daily returns (Figure 5) and 100 days moving window volatility (Figure 6) visually shows many jumps and overlapping periods of relatively high volatility.

Figure 5

CZK/EUR Exchange Rate and PX Stock Index Daily Returns
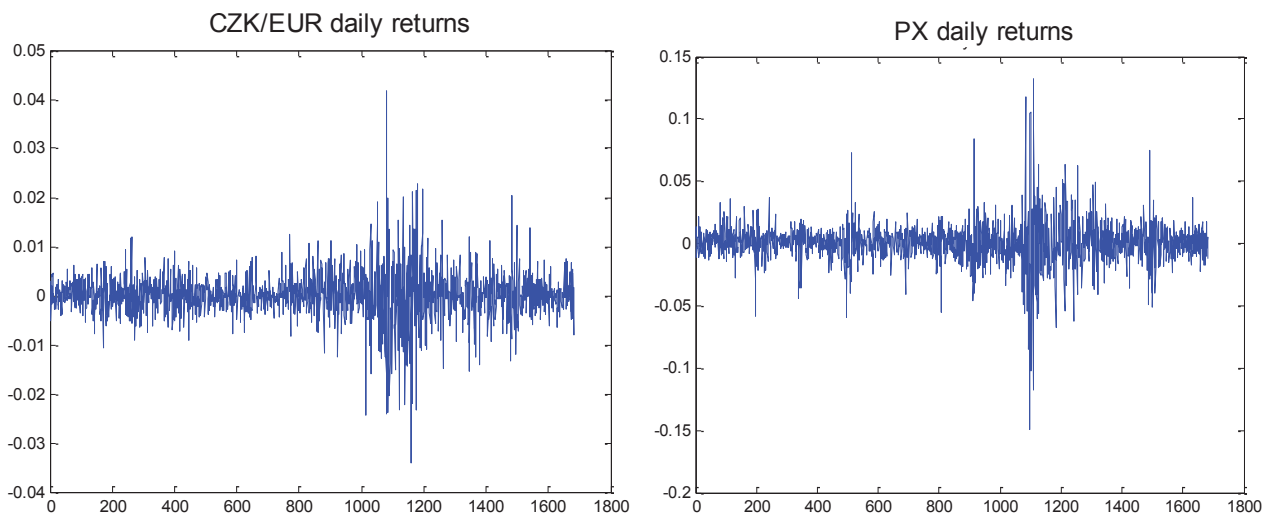

Figure 6

CZK/EUR (lower line) and PX (upper line) 100 Days Moving Window Volatility

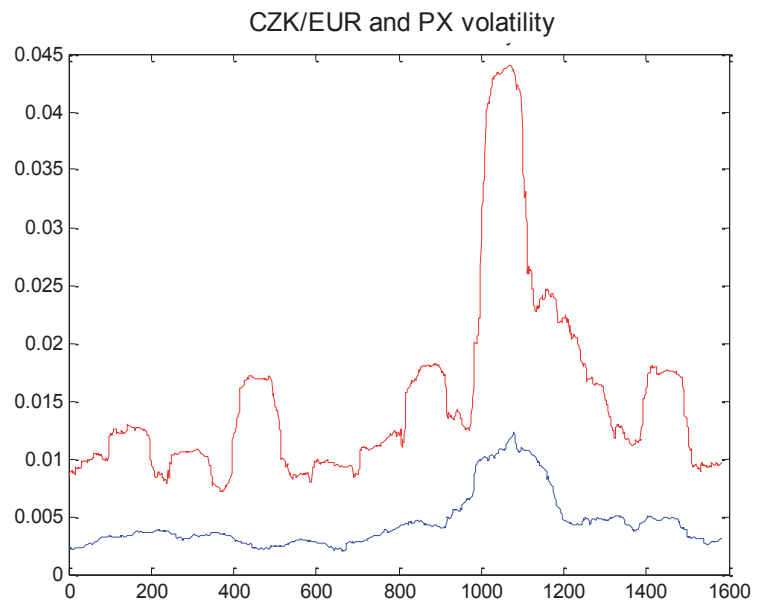

The Person's correlation of the two return series is slightly negative $-1.21 \%$ with $\mathrm{p}$-value 0.62 , i.e. if we assumed normality than we conclude the correlation does not significantly differ from zero. However, standard tests, e.g. Jarque-Bera test, reject normality of both series at the $0.1 \%$ confidence level (Table 4 ). 
Table 4.

CZK/EUR Exchange Rate and PX Stock Index Daily Returns Descriptive Statistics

\begin{tabular}{|l|c|c|}
\hline & CZK/EUR daily returns & PX daily returns \\
\hline Mean & $1.5949 \mathrm{e}-004$ & $4.0961 \mathrm{e}-004$ \\
\hline Standard deviation & 0.0049 & 0.0170 \\
\hline Skewness & 0.0292 & -0.1639 \\
\hline Kurtosis & 11.2200 & 15.8395 \\
\hline Jarque-Bera statistic & $4.7357 \mathrm{e}+003$ & $1.1561 \mathrm{e}+004$ \\
\hline p-value & $<0.001$ & $<0.001$ \\
\hline
\end{tabular}

It seems obvious that the normal VaR based on the assumption of normality and no correlation would underestimate the true risk of a combined CZK/EUR and PX investment. In order to improve our ability to predict the risk we are going to estimate and compare the four bivariate models described in Section 2.

\section{Model 1: Bivariate Pure Diffusion Model (2Diff)}

The simplest model we consider is the bivariate diffusion model. Table 5 shows MCMC estimated coefficients based on 3000 iterations when the first 1000 have been discarded. The results are in line with the descriptive statistics given in Table 4. The correlation between the two series does not significantly differ from zero in the context of this model.

Table 5.

Estimated Parameters for the CZK/EUR and PX Pure Diffusion Bivariate Model

\begin{tabular}{|l|c|c|}
\hline & \multicolumn{1}{|c|}{$\mu_{k}$} & $\sigma_{k}$ \\
\hline FX returns $(k=1)$ & $1.5345 \mathrm{e}-004(1.2927 \mathrm{e}-004)$ & $0.0049(8.6122 \mathrm{e}-005)$ \\
\hline PX returns $(k=2)$ & $4.1621 \mathrm{e}-004(4.5433 \mathrm{e}-004)$ & $0.0172(2.6047 \mathrm{e}-004)$ \\
\hline$\rho$ & $-0.0109(0.0265)$ & \\
\hline
\end{tabular}

\section{Model 2: Bivariate Jump-diffusion Model (2JD)}

Table 6 shows MCMC estimates of the bivariate jump-diffusion model with correlated jumps, but with constant volatilities. We have again used 3000 MCMC simulations, non-informative priors, and discarded the first 1000 ones. The initial means and correlations were set to 0 , initial diffusion volatilities $\sigma$ to 0.01 and jump standard deviations $\sigma_{j}$ to 0.1 . Figure 7 shows relatively fast convergence of the jump correlation $\rho_{j}$ to a surprisingly high level over $50 \%$ and the simulated kernel smoothed density. Note that the diffusion correlation $\rho$ and the jump-size correlation $\rho_{Z}$ do not significantly differ from 0 . 
Table 6

Estimated Parameters for the CZK/EUR and PX Jump-diffusion Models with Constant Volatility

\begin{tabular}{|c|c|c|c|c|}
\hline \multicolumn{5}{|c|}{ CZK/EUR jump-diffusion process } \\
\hline$\mu_{1}$ & $\sigma_{1}$ & $\lambda_{1}$ & $\mu_{1, J}$ & $\sigma_{1, J}$ \\
\hline $\begin{array}{c}2.2486 \mathrm{e}-004 \\
(9.8678 \mathrm{e}-005)\end{array}$ & $\begin{array}{c}0.0030 \\
(1.0420 \mathrm{e}-004)\end{array}$ & $\begin{array}{c}0.1737 \\
(0.0281)\end{array}$ & $\begin{array}{c}-2.9636 \mathrm{e}-004 \\
(7.1327 \mathrm{e}-004)\end{array}$ & $\begin{array}{c}0.0095 \\
(8.3013 \mathrm{e}-004)\end{array}$ \\
\hline \multicolumn{5}{|c|}{ PX jump-diffusion process } \\
\hline$\mu_{2}$ & $\sigma_{2}$ & $\lambda_{2}$ & $\mu_{2, J}$ & $\rho_{z}$ \\
\hline $\begin{array}{c}9.9833 \mathrm{e}-004 \\
(3.1909 \mathrm{e}-004)\end{array}$ & $\begin{array}{c}0.0101 \\
(3.3542 \mathrm{e}-004)\end{array}$ & $\begin{array}{c}0.1549 \\
(0.0244)\end{array}$ & $-0.0038(0.0025)$ & $0.0353(0.0027)$ \\
\hline \multicolumn{5}{|c|}{ Correlations } \\
\hline$\rho$ & $\rho_{J}$ & $\rho_{z}$ \\
\hline \multicolumn{6}{|c|}{$\rho$} & $0.5401(0.0675)$ & $0.0086(0.0896)$ \\
\hline
\end{tabular}

Figure 7

Convergence and the Bayesian Density of the Jump Correlation $\rho_{J}$
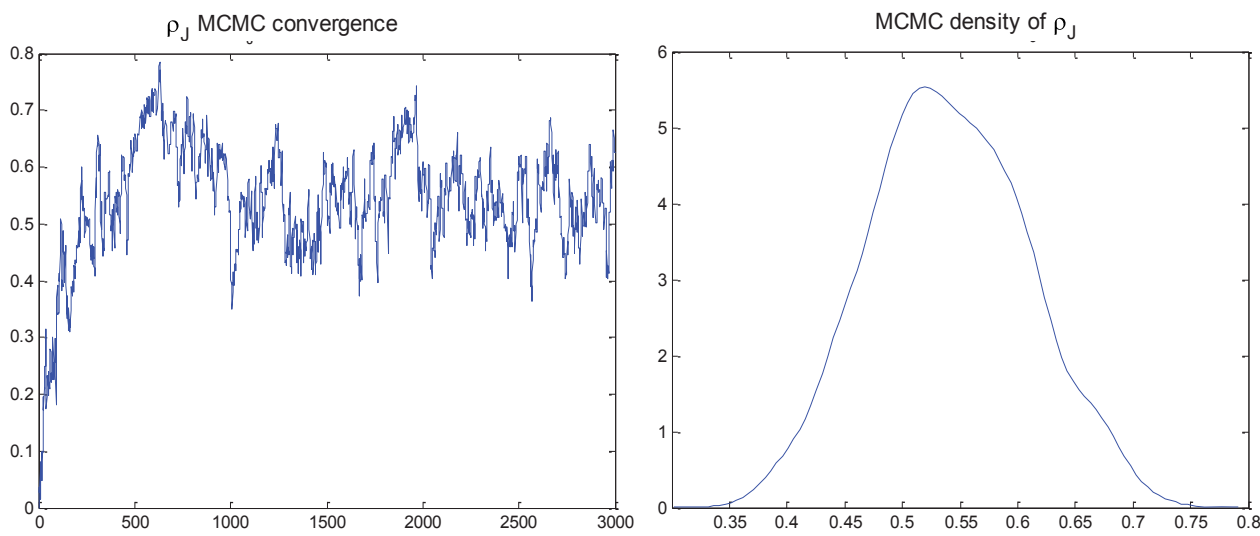

Probably the most interesting finding is that the probabilities of jumps $\lambda$ are relatively high, over $15 \%$ for both return series. However, the outcome should not be so surprising looking at the very high kurtosis (Table 4) of both series. The jump-diffusion model in fact decomposes the distribution into a mix of two normal distributions, one with the lower standard deviation $\sigma_{J}$ and the other with a higher (more than three times) standard deviation $\sigma_{J}$. While CZK/EUR mean jump size does not significantly differ from zero, the mean PX jump size is negative showing that the stock index tends to jump down rather than up, as one would expect (and as indicated by the negative skewness shown in Table 4).

The high probabilities of jumps contradict to our intuition of jumps being rare events. The MCMC estimations give us also simulated distributions of jump occurrences and jumps sizes that allow us to analyze the jumps in more detail. Each run of the MCMC 
simulation samples specific jumps times and jump sizes. Hence for each day we may calculate the empirical probability of jump and identify days where "a jump probably happened", i.e. where the probability of jump is larger than $50 \%$. For those days it makes sense to inspect the mean value of the simulated jump sizes (conditional on the jump occurrence). The results are shown in Figure 8. It is obvious that the jumps are not distributed evenly being clustered especially in the financial crises period. We will see that the jump clustering is essentially filtered out in the model with stochastic volatility.

\section{Figure 8}

\section{CZK/EUR and PX Returns Jump Probabilities and Mean Jump Sizes}
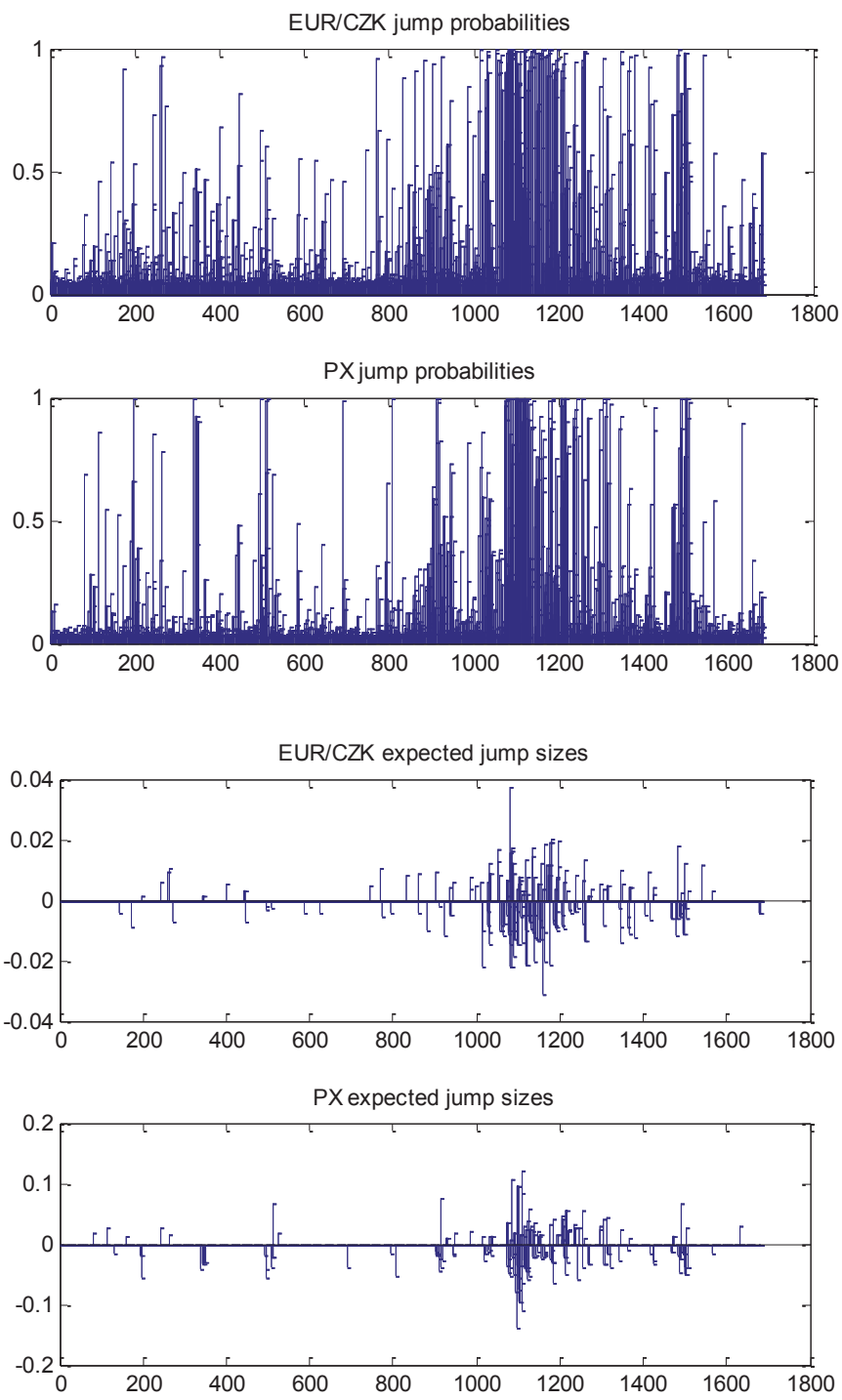


\section{Model 3: Bivariate Jump-diffusion with Stochastic Volatility (2JD SV)}

Before estimating the bivariate stochastic volatility model we firstly implement the univariate model for both return series in order to inspect the relationship between the two latent stochastic volatilities time series. The estimated parameters and their standard deviations based on $3000 \mathrm{MCMC}$ simulations are shown in Table 7. Figure 9 shows for the sake of illustration relatively fast convergence of the coefficient $\beta$ in case of the PX return process. The parameter means and standard deviations in Table 7 are based on the last 2500 simulations discarding the first 500 . Note that by introducing the stochastic volatility into the model the probabilities of jumps have been significantly reduced to less than $3 \%$ and the jump size standard deviation went up. The high value of the stochastic volatility (log-variance) autocorrelation coefficient $\beta$, almost $99 \%$ for CZK/EUR and almost $98 \%$ for PX, shows a high persistence of stochastic volatilities that is in line with other empirical studies on US stock market data (e.g. Jacquier et al., 1994, Eraker et al., 2003). The volatility of the stochastic volatility, i.e. the coefficient $\gamma$, around $13 \%$ for CZK/EUR and over $21 \%$ for PX, is also in the range estimated on US data.

Table 7

Estimated Parameters for the CZK/EUR and PX Univariate Jump Diffusion Models with Stochastic Volatility

\begin{tabular}{|c|c|c|c|c|c|c|}
\hline \multicolumn{7}{|c|}{ CZK/EUR univariate jump-diffusion process with stochastic volatility } \\
\hline$\mu_{1}$ & $\lambda_{1}$ & $\mu_{1, J}$ & $\sigma_{1, J}$ & $\alpha_{1}$ & $\beta_{1}$ & $V_{1}$ \\
\hline $\begin{array}{l}1.8506 \mathrm{e}-004 \\
(6.374 \mathrm{e}-005)\end{array}$ & $\begin{array}{l}0.0284 \\
(0.0083)\end{array}$ & $\begin{array}{l}-2.2616 \mathrm{e}- \\
004(0.0024)\end{array}$ & $\begin{array}{l}0.0117 \\
(0.0018)\end{array}$ & $\begin{array}{l}-0.1205 \\
(0.0545)\end{array}$ & $\begin{array}{l}0.9893 \\
(0.0048)\end{array}$ & $\begin{array}{l}0.1313 \\
(0.0193)\end{array}$ \\
\hline \multicolumn{7}{|c|}{ PX univariate jump-diffusion process with stochastic volatility } \\
\hline$\mu_{2}$ & $\lambda_{2}$ & $\mu_{2, J}$ & $\sigma_{2, J}$ & $\alpha_{2}$ & $\beta_{2}$ & $\gamma_{2}$ \\
\hline $\begin{array}{l}0.0012 \\
(1.921 \mathrm{e}-004)\end{array}$ & $\begin{array}{l}0.0237 \\
(0.0068)\end{array}$ & $\begin{array}{l}0.0011 \\
(0.0079)\end{array}$ & $\begin{array}{l}0.0427 \\
(0.0066)\end{array}$ & $\begin{array}{l}-0.1957 \\
(0.0613)\end{array}$ & $\begin{array}{l}0.9781 \\
(0.0069)\end{array}$ & $\begin{array}{l}0.2119 \\
(0.0247)\end{array}$ \\
\hline
\end{tabular}

Figure 9

Convergence and MCMC Simulated Density of the Parameter $\beta$ for PX Returns
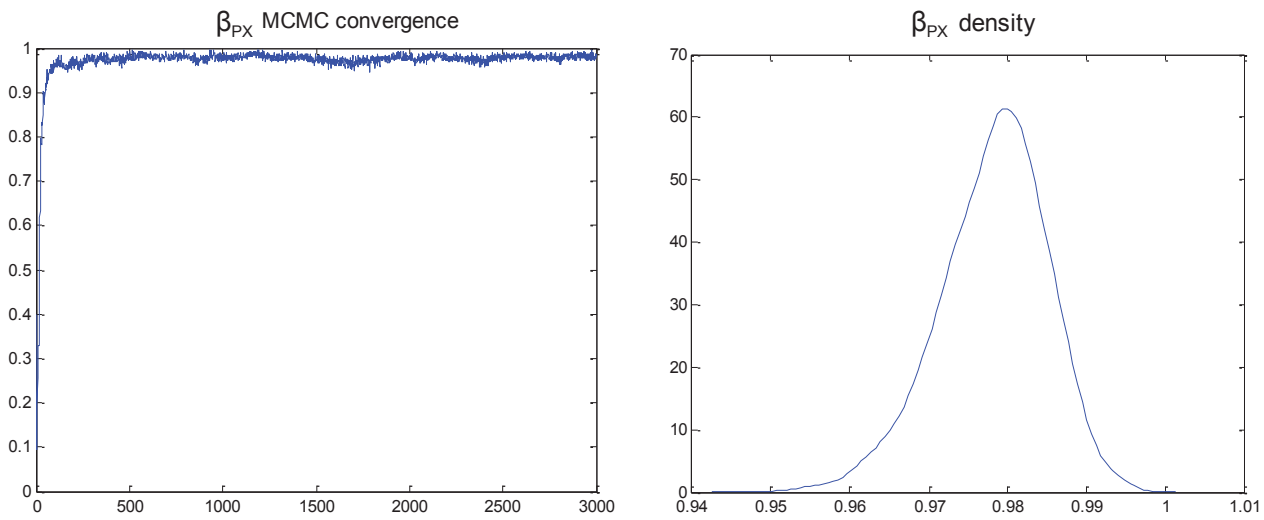
The latent stochastic volatilities are sampled at each MCMC simulation run and we get a distribution for each particular day. In order to investigate the relationship between the CZK/EUR and PX volatilities we use the mean estimates, specifically given by the equation $\hat{\sigma}_{i}=\exp \left(\overline{h_{i}} / 2\right)$, where $\overline{h_{i}}$ is the MCMC mean of normally distributed $\log V_{i}$ sampled values. Figure 10 shows that the mean stochastic volatility for both series copy well the pattern of the observed returns. The figures also explain why many jumps identified in the constant volatility model have been filtered out in the stochastic volatility model.

Figure 10

CZK/EUR and PX Returns (fine bars) and Mean Stochastic Volatilities (dark line)
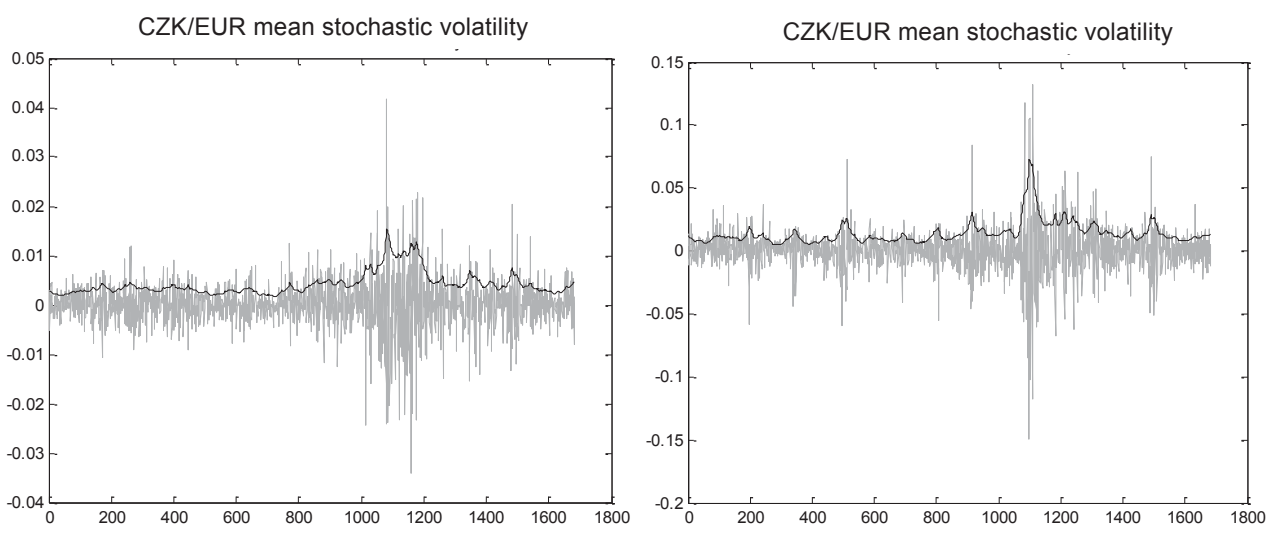

The mean stochastic volatility series and mean estimated coefficients $\alpha, \beta$ and $\gamma$ may be used to obtain the residuals of the two series $e_{F, i}^{V}$ and $e_{P X, i}^{V}$. The Pearson's correlation of the residuals is relatively low $5.7 \%$ and not significant at $1 \%$ confidence level, however, the correlation of $\bar{h}_{F X, i}$ and $\bar{h}_{P X, i}$ comes out highly significant $61.66 \%$ as indicated visually by Figure 10. Consequently, having parsimony in mind, it is reasonable to specify the bivariate jump-diffusion model with correlated stochastic volatilities just through the mutual Granger causality and without correlated volatility residuals, i.e. according to the model (23).

The model is estimated by the methodology that has been outlined in Section 2.4 and the resulting estimates are shown in Table 8 . We have run again 3000 simulations, used the last 2000 , and discarded the first 1000 . Figure 11 shows relatively fast convergence of the interesting coefficient $\beta_{21}$. The significant positive value 0.035 proves there is a Granger dependence of the PX stochastic volatility on the CZK/EUR stochastic volatility. On the other hand, the coefficient $\beta_{12}$ reflecting the causality in the opposite direction turns out not to be significant. This corresponds to our intuition: the FX market is more liquid and closely linked to the global markets while the Czech stock market liquidity is relatively low and in a sense behind the global FX market. It can be verified that the VAR(1) process estimated coefficients imply a high Pearson's correlation around 58\% close to our finding based on the univariate models. 
Table 8

Estimated Parameters for the Bivariate Jump Diffusion Models with Granger Related Stochastic Volatilities

\begin{tabular}{|c|c|c|c|c|c|c|c|}
\hline \multicolumn{7}{|c|}{ CZK/EUR jump-diffusion process with stochastic volatility } \\
\hline$\mu_{1}$ & $\lambda_{1}$ & $\mu_{1, J}$ & $\sigma_{1, J}$ & $\alpha_{1}$ & $\beta_{11}$ & $\beta_{12}$ & $\gamma_{1}$ \\
\hline $1.5188 \mathrm{e}-004$ & 0.0129 & $-9.18 \mathrm{e}-004$ & 0.0127 & -0.2694 & 0.9692 & 0.0086 & 0.2112 \\
$(5.994 \mathrm{e}-005)$ & $(0.0057)$ & $(0.0057)$ & $(0.0016)$ & $(0.0972)$ & $(0.0125)$ & $(0.0087)$ & $(0.0316)$ \\
\hline \multicolumn{7}{|c|}{ PX jump-diffusion process with stochastic volatility } \\
\hline$\mu_{2}$ & $\lambda_{2}$ & $\mu_{2, J}$ & $\sigma_{2, J}$ & $\alpha_{2}$ & $\beta_{21}$ & $\beta_{22}$ & $\gamma_{2}$ \\
\hline 0.0013 & 0.0117 & -0.0199 & 0.0447 & 0.0220 & 0.0350 & 0.9582 & 0.2268 \\
$(1.809 \mathrm{e}-004)$ & $(0.0048)$ & $(0.0143)$ & $(0.0060)$ & $(0.0827)$ & $(0.0092)$ & $(0.0078)$ & $(0.0174)$ \\
\hline \multicolumn{7}{|c|}{ Correlations } \\
\hline \multicolumn{70}{|c|}{$0.2638(0.1927)$} \\
\hline
\end{tabular}

Figure 11

MCMC Convergence and Density of the Coefficient Reflecting the Granger Dependence of the PX Stochastic Volatility on the CZK/EUR Stochastic Volatility
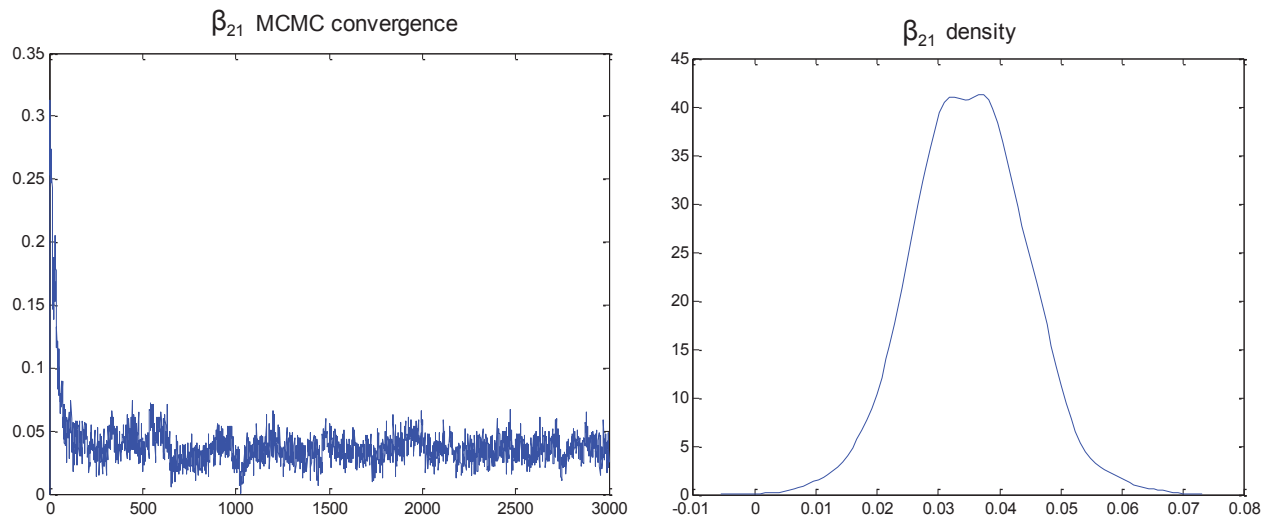

Finally we may inspect the behaviour of jumps. It is interesting to note that the jump probability fell to $1.3 \%$ in case of CZK/EUR and $1.2 \%$ in case of PX returns. Figure 12 shows the MCMC empirical daily jump probabilities. For both series there is only one day with jump probability higher than $50 \%$ and a few days with jump probabilities over $20 \%$. The stochastic volatility component has clearly filtered out the clustering and the jumps seem to be distributed evenly. There is still a positive jump occurrence correlation 0.26 and it is interesting to note that in this case the jump size correlation is positive 0.47 (though not highly significant) meaning that if there is a coincidence of jumps on both markets than the jumps probably go in the same direction. 
Looking at the jump analysis in the jump-diffusion models with and without stochastic volatility it is obvious that jump identification strongly depends on the stochastic model chosen.

Figure 12

CZK/EUR and PX Returns Jump Probabilities in the Context of the Bivariate Stochastic Volatility Model
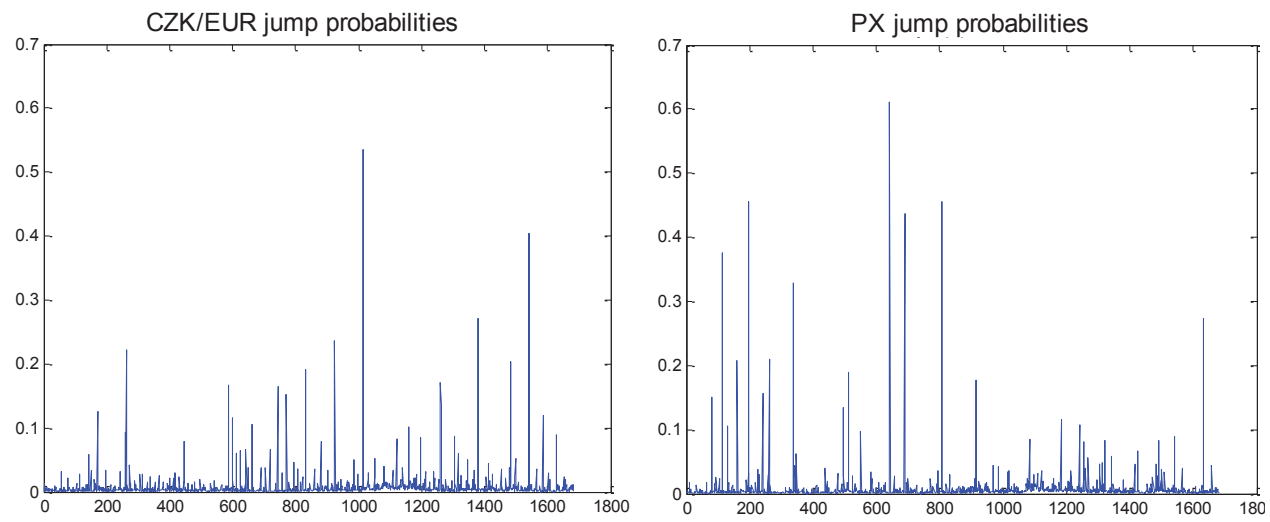

\section{Model 4: Bivariate Diffusion Model with Stochastic Volatility (2SV)}

Since the estimated jump probabilities in the previous models have been low and not highly significant Table 9 gives, for the sake of completes, the estimates of the bivariate stochastic volatility model without jumps. It is interesting to note that the estimated coefficients do not differ significantly from the results in Table 8 (with jump parameters missing).

Table 9

Estimated Parameters for the Bivariate Stochastic Volatility Model without Jumps

\begin{tabular}{|c|c|c|c|c|c|c|c|}
\hline \multicolumn{8}{|c|}{ diffusion process FX with stochastic volatility } \\
\hline$\mu_{1}$ & $\lambda_{1}$ & $\mu_{1, J}$ & $\sigma_{1, J}$ & $\alpha_{1}$ & $\beta_{11}$ & $\beta_{12}$ & $V_{1}$ \\
\hline $\begin{array}{c}1.7474 \mathrm{e}-004 \\
(5.2611 \mathrm{e}-004)\end{array}$ & - & - & - & $\begin{array}{l}-0.1981 \\
(0.0951)\end{array}$ & $\begin{array}{c}0.9791 \\
(0.0121)\end{array}$ & $\begin{array}{c}0.0041 \\
(0.0077)\end{array}$ & $\begin{array}{r}0.1750 \\
(0.0393)\end{array}$ \\
\hline \multicolumn{8}{|c|}{ diffusion process $\mathrm{PX}$ with stochastic volatility } \\
\hline$\mu_{2}$ & $\lambda_{2}$ & $\mu_{2, J}$ & $\sigma_{2, J}$ & $a_{2}$ & $\beta_{21}$ & $\beta_{22}$ & $V_{2}$ \\
\hline $\begin{array}{c}0.0013 \\
(0.0013)\end{array}$ & & & & $\begin{array}{l}-0.0111 \\
(0.0867)\end{array}$ & $\begin{array}{c}0.0387 \\
(0.0100)\end{array}$ & $\begin{array}{c}0.9501 \\
(0.0105)\end{array}$ & $\begin{array}{c}0.2564 \\
(0.0250)\end{array}$ \\
\hline \multicolumn{8}{|c|}{ Correlations } \\
\hline \multicolumn{2}{|c|}{$\rho$} & \multicolumn{3}{|c|}{$\rho_{J}$} & \multicolumn{3}{|c|}{$\rho_{z}$} \\
\hline \multicolumn{2}{|c|}{$-0.0371(0.0257)$} & \multicolumn{3}{|c|}{-} & \multicolumn{3}{|c|}{-} \\
\hline
\end{tabular}




\subsection{Comparison of the Models}

The four estimated models can be compared using the deviance information criterion (DIC) measure shown in Table 10. The last two columns give the goodness-of-fit and the model complexity measures. For example, in case of the bivariate diffusion model $p_{D}$ equals to 5 (after rounding to units), i.e. to the number of the parameters estimated, as expected. It is more difficult to interpret the $p_{D}$ values in case of the other models. The high complexity of the Model 2 (2JD) could be explained by existence of many jumps where the size needs to be in addition estimated. The high value of $p_{D}$ for the Model 4 (2SV) compared to the Model 3 (2JD SV) is, however, slightly puzzling.

An absolute difference in DIC over 10 is already considered important (Spiegelhalter et al, 2002, Asai et al., 2006), and so the models with jumps and/or stochastic volatility strongly outperform, in terms of DIC, the pure diffusion model. The best ranked model is the Model 2 (2JD) followed by the Model 3 (2JD SV). The difference between the DIC of 2JD and 2JDSV might be disappointing from the perspective of the stochastic volatility modeling and the estimation effort. However, the 2JD model estimates many jump sizes significantly improving the goodness-of-fit with respect to the estimation dataset, but the jump sizes estimates do not have any added value for predictions of future returns (distributions). This is not the case of the 2JDSV model where the last estimated stochastic volatility is used to predict future volatilities and return distributions.

The ability of the different models to predict future distributions (in particular VaR) could be compared using a back-testing procedure. This is unfortunately unfeasible at the moment since one MCMC estimation takes at least a few hours on a relatively powerful desk-top computer and back-testing would require repeating the procedure hundreds or thousands of times.

Table 10

Deviance Information Criterion of the Four Estimated Bivariate Models

\begin{tabular}{|l|c|c|c|}
\hline & $\boldsymbol{D I C}$ & $\overline{\boldsymbol{D}}$ & $\boldsymbol{p}_{\boldsymbol{D}}$ \\
\hline Model 1 (2Diff) & -22019 & -22024 & 5 \\
\hline Model 2 (2JD) & -25294 & -25479 & 185 \\
\hline Model 3 (2JD SV) & -24369 & -24430 & 61 \\
\hline Model 4 (2SV) & -24068 & -24228 & 160 \\
\hline
\end{tabular}

\section{VaR Estimations}

We are going to show VaR estimations given by the four tested models and by a benchmark model to illustrate importance of the model choice. Table 11 provides a comparison of the estimated VaR measures for the sum of lognormal returns $r_{C Z K / E U R}+r_{P X}$ calculated using the different models and by the methodology described in Section 2.5. The returns could be interpreted for example as returns on a PX stock index investment from the 
perspective of a EUR based investor as of February 2, 2011. The VaR measure has been calculated in 1, 10, and 30 days horizon, on the $95 \%$ and $99 \%$ probability level, and conditional on the point parameter estimates (Table 5 -Table 9 and the least mean variance for the SV models) or on the corresponding MCMC parameter distributions. The last line gives EWMA VaR estimates used frequently in practice, for the sake of comparison with our models. It is based on exponentially weighted moving average (EWMA) covariance matrix with the weight set at 0.97 . The number of Monte Carlo simulations has been set to 40000 .

Since the jump-diffusion (2JD) model is using constant volatilities, it is comparable rather to the diffusion model (2Diff), corresponding to a standard normal VaR estimate. On the other hand, the jump-diffusion model with stochastic volatility (2JDSV) starts with the last estimated volatility (as of February 2, 2011) and so it can be meaningfully compared rather against the benchmark EWMA model that works a recent volatility trend.

The 2JD VaR does not differ significantly from the 2Diff VaR on the $95 \%$ probability level, but, not surprisingly, the difference is more significant on the $99 \%$ probability level and in the short 1- day horizon. Over longer time horizons the jumps mix better with the normal returns and the two approaches become closer. In case of the jump-diffusion model with stochastic volatility we observe the opposite. The 2JDSV and 2SV VaR is similar to EWMA VaR in the 1-day horizon, but the difference becomes more pronounced in longer time horizon due to the stochastic volatility effect. The 30 days JDSV and $2 \mathrm{SV}$ VaR on the $99 \%$ probability level exceeds the EWMA VaR by almost $40 \%$.

It should be pointed that no definite conclusion can be drawn from the differences in VaR estimates. Nevertheless, if the true process was a pure diffusion one, then the choice of the model should not matter. Significant parameters of the models with jumps and stochastic volatilities, the goodness-of-fit comparison, and the different VaR values demonstrate that the choice of model is important.

Table 11

Comparison of Value at Risk in the 1, 10, or 30 Day Horizon, Based on Point Parameter Estimates /Bayes Simulation, and on the 95\%, 99\% Probability Level Estimated Using the Different Models

\begin{tabular}{|l|l|l|l|l|l|l|}
\hline $\begin{array}{l}\text { VaR / Bayes } \\
\text { VaR }\end{array}$ & $\mathbf{( 1 , 9 5 \% )}$ & $\mathbf{( 1 , 9 9 \% )}$ & $\mathbf{( 1 0 , 9 5 \% )}$ & $\mathbf{( 1 0 , 9 9 \% )}$ & $\mathbf{( 3 0 , 9 5 \% )}$ & $\mathbf{( 3 0 , 9 9 \% )}$ \\
\hline $\begin{array}{l}\text { Model 1 } \\
\text { (2Diff) }\end{array}$ & $0.0299 /$ & $0.0421 /$ & $0.0985 /$ & $0.1361 /$ & $0.1780 /$ & $0.2451 /$ \\
\hline Model 2 & 0.0297 & 0.0417 & 0.0986 & 0.1361 & 0.1787 & 0.2433 \\
(2JD) & 0.0242 & $0.0520 /$ & $0.0969 /$ & $0.1414 /$ & $0.1743 /$ & $0.2437 /$ \\
\hline Model 3 & 0.0177 & 0.02632 & 0.0962 & 0.1405 & 0.1781 & 0.2480 \\
(2JDSV) & 0.0179 & 0.0284 & 0.0654 & 0.1041 & 0.1272 & 0.1957 \\
\hline Model 4 & $0.0213 /$ & $0.0293 /$ & $0.0763 /$ & $0.1040 /$ & $0.1499 /$ & $0.2048 /$ \\
(2SV) & 0.0214 & 0.0300 & 0.0769 & 0.1086 & 0.1505 & 0.2092 \\
\hline EWMA & 0.0185 & 0.0262 & 0.0586 & 0.0828 & 0.1014 & 0.1435 \\
\hline
\end{tabular}




\section{Conclusion}

We have proposed and tested a bivariate jump-diffusion model with stochastic volatilities where the jump occurrence, jump-sizes, and the volatilities are, in general, correlated. The stochastic volatility correlation is captured through the Granger causality in both directions. Our proposed MCMC estimation technique extends the one of Jacquier et al. (2007). The tests have demonstrated ability of the algorithm to recover consistently the true parameters used to generated testing data. The model has been, for the sake of empirical illustration, applied on the CZK/EUR and the Czech stock index PX data (2004-2011). The results show that there is Granger causality in stochastic volatility going from the CZK/EUR market to the Czech stock market but not vice versa. The estimated probabilities of jumps are around $1.2 \%$. The jumps and jump sizes appear to be strongly correlated. We have compared the model with the pure diffusion model, with the stochastic volatility model without jumps, and with the jump-diffusion model with constant volatility. It is interesting to note that the probabilities of jumps in the pure jump diffusion model would differ dramatically being more than $15 \%$ for both processes, if the volatility was constant. The four tested models have been compared applying the deviance information criterion (DIC) with the pure jump-diffusion and the stochastic volatility jump-diffusion models performing the best. However, it can be argued that the proposed stochastic volatility jump-diffusion model giving forward looking volatility estimates is the most appropriate for VaR calculations and generally for future returns distribution modeling.

The research can be extended in several directions: the model and the estimation technique can be applied to other time series, to high frequency data, and generalized to the general multivariate case requiring further computational efficiency improvements.

\section{Literature}

Asai, M., McAleer, M., Jun Yu, J. (2006), "Multivariate Stochastic Volatility: A Review." Econometric Reviews, 25(2-3), pp. 145-175.

Cont, R., Tankov, P. (2004), "Financial Modelling With Jump Processes." Chapman \& Hall/CRC Financial Mathematics Series, p. 535.

Eraker, B. (2004), “Do Stock Prices and Volatility Jump? Reconciling Evidence from Spot and Option Prices." The Journal of Finance, Vol. LIX, No. 3, June, pp. 1367-1403.

Eraker, B., Johannes, M., Polson, N. (2003), "The Impact of Jumps in Volatility and Returns." The Journal of Finance, Vol. LVIII, No. 3, June, pp. 1269-1300.

Heston, S. (1993), "Closed-form Solution of Options with Stochastic Volatility with Application to Bond and Currency Options." Review of Financial Studies 6, pp. 327-343.

Jacquier, E, Johannes, M., Polson, N. (2007), "MCMC Maximum Likelihood for Latent State Models." Journal of Econometrics 137, pp. 615-640.

Jacquier, E., Polson, N., Rossi, P. (1994), "Bayesian Analysis of Stochastic Volatility Models." Journal of Business \& Economic Statistics, Vol. 12, No. 4, October, pp. 69-87.

Johannes, M., Kumar, R. (1999), “State Dependent Jump Models: How Do US Equity Indices Jump?" Working Paper, Columbia University; available at http://faculty.chicagobooth.edu/ nicholas.polson/research/papers/statedep.pdf, pp. 13. 
Johannes, M., Polson, N. (2009), "MCMC Methods for Financial Econometrics." In Ait-Sahalia, Hansen, L. P., eds., Handbook of Financial Econometrics. pp. 1-72.

Lynch, S. M. (2007), Introduction to Applied Bayesian Statistics and Estimation for Social Scientists. Springer, pp. 359.

Rachev, S. T., Hsu, J. S, Bagasheva, B. S., Fabozzi, F. J. (2008), Bayesian Methods in Finance. The Frank J. Fabozzi Series, Wiley, pp. 329.

Shephard, N. (2004), Stochastic Volatility: Selected Readings. Oxford: Oxford University Press, p. 534 .

Shreve, S. E. (2004), Stochastic Calculus for Finance II / Continuous Time Models. Springer, p. 550.

Šopov, B., Seidler, J. (2010), "Yield Curve Dynamics: Regional Common Factor Model." IES Working Paper 17/2000, IES FSV, Charles University, p. 21.

Spiegelhalter, D. J., Best, N. G., Carlin, B. P., van der Linde, A. (2002), "Bayesian Measures of Model Complexity and Fit" (with discussion). Journal of the Royal Statistical Society, Series B., 64, pp. 583-639.

Yu, J., Meyer, R. (2006), "Multivariate Stochastic Volatility Models: Bayesian Estimation and Model Comparison." Econometric Reviews, 25(2-3), pp. 361-384. 